\title{
System Identification and Model-Based Flight Control System Design for an Agile Maneuvering Quadrotor Platform
}

\author{
Burak Yuksek*, Emre Saldiran ${ }^{\dagger}$ and Aykut Cetin \\ Istanbul Technical University, Istanbul, Turkey, 34469 \\ Ramazan Yeniceri ${ }^{\S}$ \\ Istanbul Technical University, Istanbul, Turkey, 34469 \\ Gokhan Inalhan ${ }^{\text {II }}$ \\ Cranfield University, United Kingdom, MK43 OAL
}

\begin{abstract}
In this paper, we provide a system identification, model stitching and model-based flight control system design methodology for an agile maneuvering quadrotor micro aerial vehicle (MAV) technology demonstrator platform. The proposed MAV is designed to perform agile maneuvers in hover/low-speed and fast forward flight conditions in which significant changes in system dynamics are observed. As such, these significant changes result in considerable loss of performance and precision using classical hover or forward flight model based controller designs. To capture the changing dynamics, we consider an approach which is adapted from the full-scale manned aircraft and rotorcraft domain. Specifically, linear mathematical models of the MAV in hover and forward flight are obtained by using the frequency-domain system identification method and they are validated in time-domain. These point models are stitched with the trim data and quasi-nonlinear mathematical model is generated for simulation purposes. Identified linear models are used in a multi-objective optimization based flight control system design approach in which several handling quality specifications are used to optimize the controller parameters. Lateral reposition and longitudinal depart/abort mission task elements from ADS-33E-PRF are scaled-down by using kinematic scaling to evaluate the proposed flight control systems. Position hold, trajectory tracking and aggressiveness analysis are performed, Monte-Carlo simulations and actual flight test results are compared. The results show that the proposed methodology provides high precision and predictable maneuvering control capability over an extensive speed envelope in comparison to classical control techniques. Our current work focuses on i) extension of the flight envelope of the mathematical model and ii) improvement of agile maneuvering capability of the MAV.
\end{abstract}

\section{Introduction}

In recent years, urban air mobility (UAM) has an increasing demand especially for people and cargo transportation in the urban environment in which strict requirements should be defined because of its dense structure. Agents in this environment should be operated with effective coordination to ensure the airspace safety with increased versatility, speed, and minimum environmental impact. In this work, we provide a system identification, model stitching and model-based flight control system design methodology for an agile maneuvering technology demonstrator quadrotor platform. The proposed methodology is to ensure high precision maneuvering control capability over an extensive speed envelope in comparison to classical control techniques.

Novel manned and unmanned aerial vehicle applications (such as CityAirbus, Vahana and Volocopter) have a promising role in the future of the urban air mobility and cargo services (Skyways, Uber Air and Prime Air) which are aimed to provide sustainable solutions with minimum infrastructure requirements. However, integration of the

\footnotetext{
*Ph.D. Candidate, ITU Aerospace Research Center, yuksekb@itu.edu.tr, AIAA Student Member

${ }^{\dagger}$ M.Sc. Student, ITU Aerospace Research Center, saldiran@itu.edu.tr

${ }^{\ddagger}$ Research Associate, ITU Aerospace Research Center, cetinayk@itu.edu.tr.

$\S$ Assist. Professor, ITU Department of Aeronautical Engineering, yenicerir@itu.edu.tr.

IProfessor, School of Aerospace, Transport and Manufacturing / Centre for Autonomous and Cyber-Physical Systems, inalhan@cranfield.ac.uk, AIAA Associate Fellow
} 


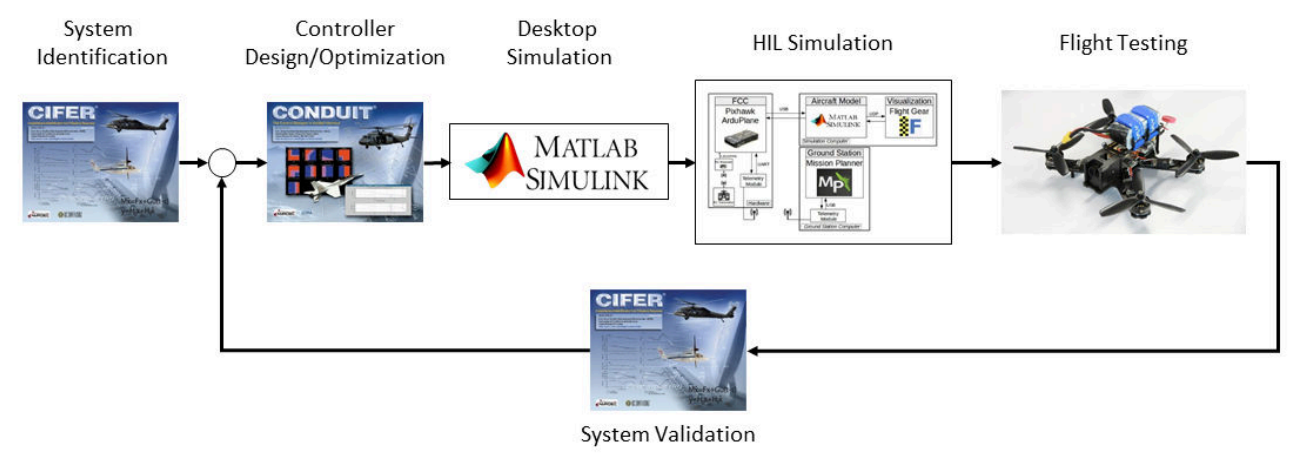

Fig. 1 General view of the desktop-to-flight design workflow [1].

manned/unmanned vehicles into the urban airspace is a key challenge in the urban air mobility concept. Specifically, predictability of the dynamic behaviour of these vehicles is crucial in ensuring Unmanned Aircraft System Traffic Management (UTM) solutions which is capable of accommodating all of these vehicles in the same airspace safely and efficiently.

Blending with the general Air Traffic Management (ATM), it is obvious that the aerial vehicles integrated into the airspace should have mid/high fidelity mathematical model and adequate control system performance for hover, low speed, and fast forward flight phases. At this point, accurate/verified mathematical modeling and proper/transparent control system design process is crucial to obtain a certifiable flight control/autonomy system.

In literature, there are two fundamental methods for modeling an aerial vehicle. The first method is called as physics-based modeling approach in which it is required to calculate the aerodynamic, inertial and structural parameters by using analytical and empirical tools. This approach can be used before the aircraft has been built and it is useful to gain preliminary insights about the dynamics of the designed aircraft. However, it can be time intensive to obtain these parameters by using several test setups and analytical/empirical tools. The second approach for modeling of the aerial platform is system identification method which can be applied in time-domain and frequency-domain. In this process, several pre-designed flight tests are performed and aerodynamic parameters are obtained by using the recorded responses of the aircraft. The system identification process can also be utilized to quantify the difference in the actual and predicted responses which is useful to improve the mathematical model fidelity of the aerial vehicle. In other words, physics-based modeling and system identification tools can be used in a complementary way.

Control system design process for the aerial vehicles requires to consider lots of handling quality specifications to provide mission safety and reliability. The importance of these requirements is increased significantly for the missions performed in the urban airspace. One of the first issue to maintain the flight safety is designing a control system which has adequate stability characteristics and reference signal tracking performance. Hence, several handling quality requirements are developed to design and evaluate the flight control systems. For manned fixed-wing and rotary-wing aircrafts, these requirements are collected in several handbooks such as MIL-STD-1797B [2] and ADS-33E-PRF [3], respectively.

Methodological design process and a verified mathematical model are key elements in flight control system design applications. Hence, in this work, an iterative design pathway which is known as desktop-to-flight control system design workflow [1], is utilized. This methodology is developed and validated by several applications on the manned aerial systems [1]. In this workflow, system identification, multi-objective optimization based flight control system design, desktop simulations, hardware-in-the-loop tests and validation/verification steps are tightly connected in an iterative way. The general scheme of the desktop-to-flight design workflow is given in Fig.1.

In recent years, as the integration of the MAV platforms has been increasing in military and civil applications, the desktop-to-flight design workflow is adapted for the unmanned aerial vehicles to speed up the design/validation process and decrease the development costs. There are several successful implementations applied on fixed-wing and rotary-wing unmanned platforms. In [4], a longitudinal linear mathematical model of a fixed-wing unmanned aerial vehicle (UAV) is identified and pitch attitude control system is designed to achieve adequate tracking performance of a ground target by using a body-fixed camera. In [5], lateral, longitudinal and directional dynamics of Ranger-EX fixed-wing UAV are identified and control system design is performed to improve the attitude reference tracking and disturbance rejection capabilities. In [6], a bare-airframe model of a quadrotor platform is identified and attitude control 


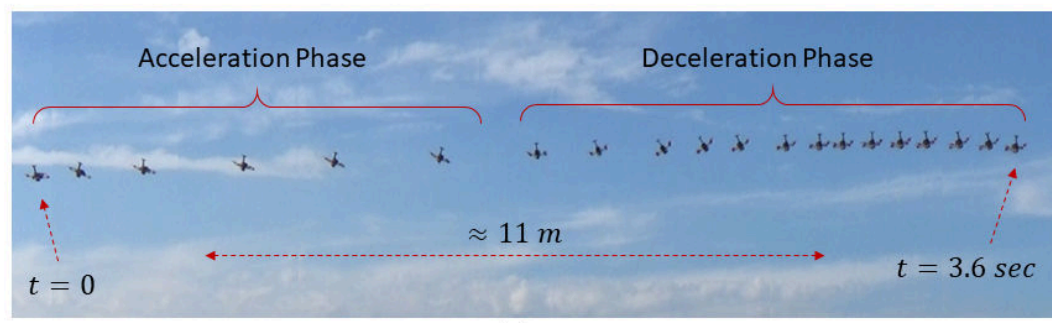

(a)

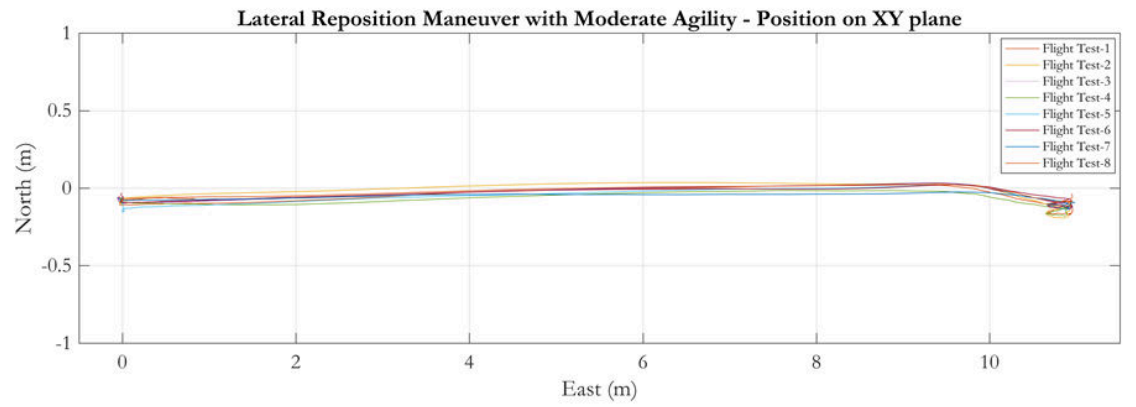

(b)

Fig. 2 Moderate agility lateral reposition maneuver. a) A footage from actual flight tests, b) Recorded North and East position.

system is designed to improve the attitude reference tracking performance. In [7], coupled longitudinal/heave axis model of a tail-sitter UAV (AeroVironment Quantix) is identified and legacy control systems are optimized to meet the stability, performance and disturbance rejection requirements.

Model fidelity level of a UAV platform plays a crucial role in formulating the efficiency airspace integration. Specifically, accurate mathematical models, covering the flight envelope of the aerial vehicle as much as possible, ensures capability to provide high performance navigation capabilities. This specific issue becomes more critical for the rotorcraft platforms because of significant deviations in the system dynamics in fast forward flight conditions. To increase the model fidelity in the forward flight phase, model stitching technique is developed by Zivan and Tischler in [8] and applied to obtain the continuous full flight envelope model of the Bell 206 helicopter. In [9], full-flight envelope mathematical model of the Calspan NF-16D Variable-stability In-flight Simulator Test Aircraft (VISTA) is developed by utilizing the stitching method and verified by using the recorded flight data. In addition to the manned aircrafts, model stitching is also applied for the UAVs and MAVs. In [10], full-flight envelope mathematical model of the unmanned K-MAX rotorcraft platform is obtained and flight control systems are optimized. In [11], model stitching method is applied for a quadrotor platform and developed STITCH software is introduced. In [12], stitched model of an octocopter platform, which performs package delivery missions with the varying size of payloads, is generated and verified.

In previous studies, system identification and controller design processes are applied for several non-agile quadrotor platforms such as 3DR Iris+. Due to the narrow flight envelope of these MAV platforms, stitched quasi-nonlinear models cover forward flight conditions within $0-10 \mathrm{~m} / \mathrm{s}$ total airspeed. However, this interval is not adequate for a racer quadrotor platform which is able to perform fast forward flight and agile maneuvers. Hence, we focused on obtaining a quasi-nonlinear model and designing suitable inner/outer loop controllers which are valid for a wide range of flight envelope.

This paper presents the application and experimental demonstration of the desktop-to-flight design workflow on a high performance agile maneuvering quadrotor platform which is capable of fast forward flight up to $32 \mathrm{~m} / \mathrm{s}$ airspeed. To the authors' knowledge, this is the first time that this methodology is extended to such a technology demonstrator based on a racer system. Linear mathematical models for hover and forward flight are obtained by utilizing the frequency-domain system identification process. Then, a quasi-nonlinear stitched mathematical model of the quadrotor is developed for hover/low speed and forward flight conditions. To obtain an adequate trajectory tracking performance, required handling qualities are determined and some of them are modified for the proposed small scale MAV by using Froude scaling analysis. Based on these selected handling qualities, inner- and outer-loop control systems of the legacy 
controller are modified and optimized to improve the stability and performance of the closed-loop system. Lateral reposition and longitudinal depart/abort maneuvers from ADS-33E-PRF are scaled-down according to kinematic scaling method. Then, several flight tests and Monte-Carlo simulations are performed to evaluate the closed-loop system performance. A footage and GPS position data from the actual flight tests are given in Fig.2.

This paper is organized as follows; in Section II, the MAV platform and subsystems are explained. In Section III, the linear model structure is given for hover and forward flight. In Section IV, frequency-domain system identification and time-domain verification processes for hover and forward flight are described. In Section V, the simulation environment is generated based on the stitched quasi-nonlinear mathematical model. In Section VI, selected handling qualities are described, attitude and trajectory tracking control systems are designed. In Section VII, simulation and flight test results are evaluated and compared. In Section VIII, concluding remarks are given and future works are explained.

\section{Experimental Platform}

A high performance racer type quadrotor platform is used to track the given trajectory because of its aggressive maneuvering capability and wide airspeed range in forward flight phase between $0-32 \mathrm{~m} / \mathrm{s}$. General view of the test platform is shown in Fig.3. To achieve a high level of thrust-to-weight ratio, carbon fiber plates are used in the airframe structure. It has EMAX RS2205 2600 KV brushless DC (BLDC) motors, Hobbywing 30A Electronic speed controller (ESC), 4S LiPo battery and $5 \times 4.5$ inch tri-blade propellers.
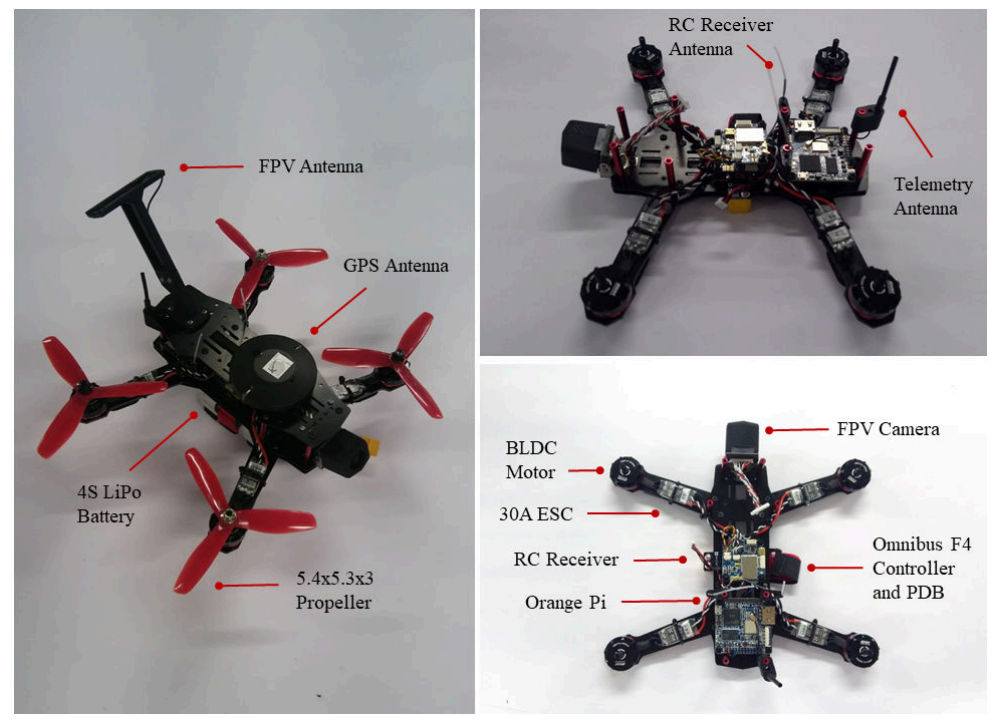

Fig. 3 General view of the high performance agile maneuvering drone platform.

In avionics structure, Omnibus F4Pro V3 and Arducopter are used as flight control system hardware and software, respectively. Omnibus F4Pro V3 is a small size and low weight flight control hardware and it is widely used in the racer quadrotor community. It has STM32F405 micro-controller, barometer, accelerometer, and gyroscope sensors onboard. Proficnc Here GPS receiver, which contains u-blox M8N GPS module and a magnetometer, is connected to the flight control board externally. In order to achieve high-frequency data logging, Arducopter flight control software runs on the Chibios which is a small footprint Real-Time Operating System (RTOS).

To increase telemetry range and allow high-speed communication between the MAV and ground station, Orange Pi Zero Plus2, which is a small size computer with the embedded WiFi module, is used on-board. Orange Pi and Omnibus flight control system is connected with the UART serial communication protocol. General scheme of the avionics structure is shown in Fig.4.

In the next section, mathematical model structure, which includes linear and angular accelerations, Euler rates and propulsion system dynamics, is introduced. 


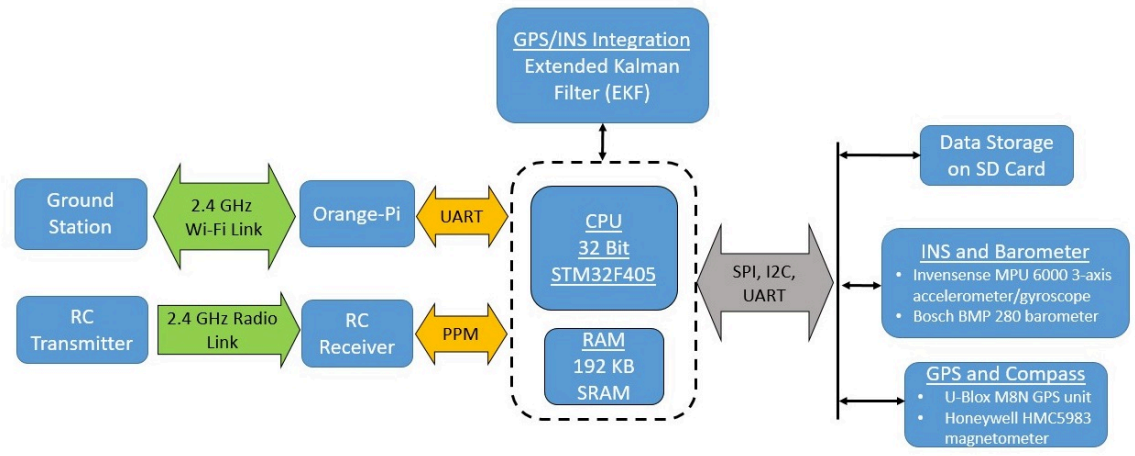

Fig. 4 General scheme of the Racer drone avionics architecture.

\section{Mathematical Model Structure}

As a preliminary step for the frequency-domain system identification process, it is important to define a linear mathematical model structure for hover and forward flight conditions. The general structure of the linearized 6 degree-of-freedom (DoF) equations of motion (EoM) in state-space form is given in Eq. (1).

$$
\begin{aligned}
\mathbf{M} \dot{\mathbf{x}} & =\mathbf{F} \mathbf{x}+\mathbf{G u}(t-\tau) \\
\mathbf{y} & =\mathbf{H}_{\mathbf{0}} \mathbf{x}+\mathbf{H}_{\mathbf{1}} \mathbf{x}
\end{aligned}
$$

where $\mathbf{x}$ is the state vector, $\mathbf{y}$ is the measurement vector, $\mathbf{F}$ includes gradients to state perturbations in trim conditions, $\mathbf{G}$ includes the gradients to control perturbations, $\tau$ is the time delay vector, $\mathbf{M}$ includes the parameters which are depend on the rates of change of the state variables, $\mathbf{H}_{1}$ and $\mathbf{H}_{0}$ matrices are called as measurement matrices and they define the measurement vector by using the states and state derivatives. State and measurements vectors are given in Eq. (2) and Eq. (3).

$$
\begin{gathered}
\mathbf{x}^{T}=\left[\begin{array}{llllllllllll}
u & v & w & p & q & r & \phi & \theta & \psi & \delta_{a}^{\prime} & \delta_{e}^{\prime} & \delta_{r}^{\prime}
\end{array}\right] \\
\mathbf{y}=\left[\begin{array}{llllllllll}
\dot{u} & \dot{v} & \dot{w} & p & q & r & a_{x} & a_{y} & a_{z}
\end{array}\right]
\end{gathered}
$$

Here, $\dot{u}, \dot{v}, \dot{w}$ cannot be measured directly. So, they should be reconstructed by using the accelerometer measurement definition as shown in Eq. (4).

$$
\begin{aligned}
& a_{x}=\dot{u}+W_{0} q-V_{0} r+g \cos \left(\Theta_{0}\right) \theta+z_{a} \dot{q}-y_{a} \dot{r} \\
& a_{y}=\dot{v}+U_{0} r-W_{0} p-g \cos \left(\Theta_{0}\right) \phi-z_{a} \dot{p}+x_{a} \dot{r} \\
& a_{z}=\dot{w}+V_{0} p-U_{0} q+g \sin \left(\Theta_{0}\right) \theta+y_{a} \dot{q}-x_{a} \dot{q}
\end{aligned}
$$

where $\left[x_{a}, y_{a}, z_{a}\right]$ defines the offset between the center of gravity and inertial measurement unit (IMU). In the technology demonstrator quadrotor platform, the center of gravity is quite close to the IMU location and these offsets can be neglected.

The actuation system dynamics (ESC, motor and propeller assembly) on roll and pitch axes are modelled as a first-order differential equation as shown in Eq. (5).

$$
\dot{\delta}^{\prime}=\omega\left(\delta_{c m d}-\delta^{\prime}\right)
$$

where, $\delta_{c m d}, \delta^{\prime}$ and $\omega$ describe commanded input, actual output and propulsion system natural frequency for lateral, longitudinal and directional dynamics, respectively.

Unlike roll and pitch axis control moments, the quadrotor platform generates yaw rate by using differential drag moments of the propellers. As a result of this situation, phase of the $r(s) / \delta_{r}(s)$ frequency response is nearly constant in low frequency region which indicates a lead effect in this input-output pair (shown in Fig. 18 in Appendix). To identify the lead in the yaw axis of the quadrotor, yaw rate dynamics is modeled as given in Eq. (6) [12].

$$
\dot{r}+\tau_{\text {lead }} \dot{\delta}_{r}^{\prime}=N_{v} v+N_{p} p+N_{r} r+N_{\delta_{a}^{\prime}} \delta_{a}^{\prime}+N_{\delta_{r}^{\prime}} \delta_{r}^{\prime}
$$


Here, $\tau_{\text {lead }}$ is set as a flexible variable in the identification process and it is used to capture the dynamical effects of this extra zero on the yaw dynamics.

The propulsion system model is integrated into the linearized 6-DoF mathematical model in the state-space structure. The linearized 6-DoF bare-airframe dynamical model including aerodynamics, gravitational and coriolis terms can be written as shown in Eq. (7).

In the next section, system identification and verification process is performed for the agile maneuvering quadrotor platform in hover and forward flight conditions.

$$
\begin{aligned}
\dot{u} & =X_{u} u+X_{w} w+\left(X_{q}-W_{0}\right) q-g \cos \left(\Theta_{0}\right) \theta+X_{\delta_{e}} \delta_{e}^{\prime} \\
\dot{v} & =Y_{v} v+\left(Y_{p}+W_{0}\right) p+\left(Y_{r}-U_{0}\right) r+g \cos \left(\Theta_{0}\right) \phi+Y_{\delta_{a}} \delta_{a}^{\prime}+Y_{\delta_{r}} \delta_{r}^{\prime} \\
\dot{w} & =Z_{u} u+Z_{w} w+\left(Z_{q}+U_{0}\right) q-g \sin \left(\Theta_{0}\right) \theta+Z_{\delta_{e}} \delta_{e}^{\prime} \\
\dot{p} & =L_{v} v+L_{p} p+L_{r} r+L_{\delta_{a}} \delta_{a}^{\prime} \\
\dot{q} & =M_{u} u+M_{w} w+M_{q} q+M_{\delta_{e}} \delta_{e}^{\prime} \\
\tau_{l e a d} \dot{\delta}_{r}^{\prime}+\dot{r} & =N_{v} v+N_{p} p+N_{r} r+N_{\delta_{a}} \delta_{a}^{\prime}+N_{\delta_{r}} \delta_{r}^{\prime} \\
\dot{\phi} & =p+r \tan \left(\Theta_{0}\right) \\
\dot{\theta} & =q \\
\dot{\psi} & =r \sec \left(\Theta_{0}\right) \\
\dot{\delta}_{a}^{\prime} & =-\omega_{a} \delta_{a}^{\prime}+\omega_{a} \delta_{a_{c m d}} \\
\dot{\delta}_{e}^{\prime} & =-\omega_{e} \delta_{e}^{\prime}+\omega_{e} \delta_{e_{c m d}} \\
\dot{\delta}_{r}^{\prime} & =-\omega_{r} \delta_{r}^{\prime}+\omega_{r} \delta_{r_{c m d}}
\end{aligned}
$$

\section{System Identification and Verification}

One of the most challenging task in modeling and flight control system design process is obtaining a suitable bare-airframe model of the aerial vehicle which contains airframe and propulsion system (ESC, BLDC motors, propellers and mixer) dynamics. Accuracy of the mathematical model directly affects the controller performance and reduces development costs.

The frequency-domain system identification method is a powerful tool to identify the bare-airframe dynamics of the aerial vehicle. In this process, the aerial vehicle is excited on each axis by using variable frequency sinusoidal input signal which covers a specific frequency range. After performing several flight tests, recorded input-output dataset is used in Comprehensive Identification from Frequency Responses (CIFER) software to obtain frequency responses of the aerial vehicle on each axis. Then, a suitable linear mathematical model is fitted on the frequency responses and identified models are verified in time-domain by using doublet input signals [13]. The system identification and verification process and results are explained in the following subsections.

\section{A. Frequency Response Generation}

Frequency sweep flight tests are performed to excite the interested bare-airframe dynamics of the quadrotor. One of the critical issue in designing the frequency sweep input is determining the frequency range of the test signal. Because of the unstable dynamics of the rotorcrafts, frequency range of interest is determined based on the crossover frequency of the closed-loop system as shown in Eq. (8) [13].

$$
0.3 \omega_{c} \leq \omega \leq 3 \omega_{c}
$$

As an initial guess, crossover frequency values of the IRIS+ quadrotor platform are used in which $\omega_{c} \approx 20-30 \mathrm{rad} / \mathrm{s}$ for lateral and longitudinal axes and $\omega_{c} \approx 6 \mathrm{rad} / \mathrm{s}$ for directional axis [14].

According to the data collecting procedure given in [13], automated sweep signal is designed to cover 1-60 rad/s frequency range. The individual sweep record length is $90 \mathrm{sec}$ and duration of the additional hover flight at the beginning and at the end of the sweep tests is 5 seconds. The concatenated record length is 200 seconds, data sampling time and filter cut-off frequency are set as $250 \mathrm{~Hz}$ and $25 \mathrm{~Hz}$, respectively.

Because of the unstable dynamics of the quadrotor platform, frequency sweep tests should be performed while the inner-loop attitude control system is engaged. So, altitude-hold mode of the ArduCopter is used while performing the 


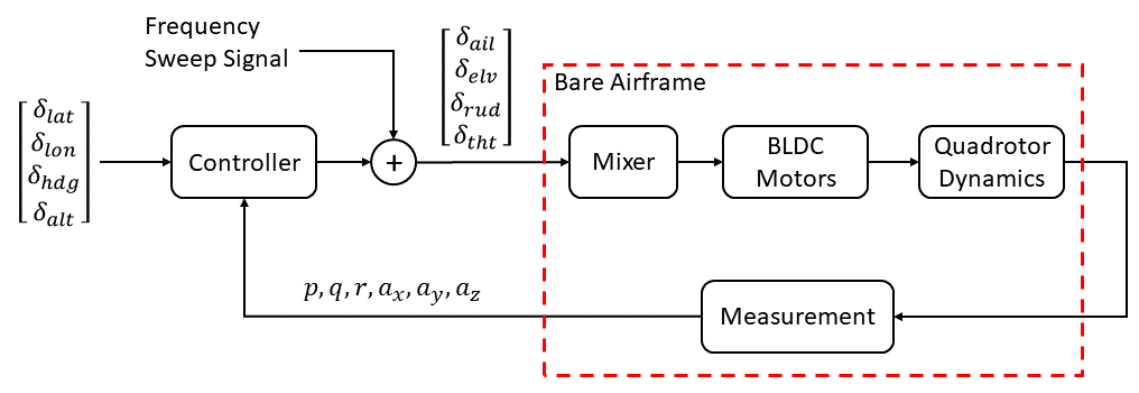

Fig. 5 Block diagram and input/output definitions for frequency sweep tests.

frequency sweep tests. To excite the bare-airframe dynamics directly, the frequency sweep test signals are summed with the controller signal and applied into the mixer as shown in Fig. 5.

After the system identification test flights, body-axis acceleration, body-axis rate and mixer inputs for lateral, longitudinal and directional axes are recorded on an SD card. A sample flight test record for the lateral axis system identification process is given in Fig. 6a.

\section{B. Obtaining the Speed Derivatives for Hover Flight Conditions}

As mentioned before, because of the unstable dynamical characteristics of the quadrotor bare-airframe, it is necessary to perform the system identification flight tests by using the inner-loop controllers of the ArduCopter. However, low frequency sweep test signal will be suppressed by the controller and this results in low coherence of the identified angular rate responses in the low frequency region. Hence, it may be difficult to identify the speed damping derivatives $\left(X_{u}, Y_{v}\right)$ and speed stability derivatives $\left(M_{u}, L_{v}\right)$. Although the coherence is low for the control inputs, there is good energy content in the $\dot{u}, \dot{v}, \theta$ and $\phi$ as a result of the kinematic consistency. So, these signals can be used to obtain the speed derivatives of the aerial platform [13]. Identified speed damping and stability derivatives in this step can be used as initial guess in the frequency-domain system identification process.

For hover conditions, lateral specific force can be given as shown in Eq. (9) in simplified form.

$$
\dot{v}=Y_{v} v+g \phi
$$

After calculating the time derivative and performing the Laplace transform, transfer function of $\dot{v}(s) / \phi(s)$ is generated as shown in Eq. (10) which allows the direct identification of the lateral speed damping derivative $Y_{v}$ from the roll axis sweep flight test data.

$$
\frac{\dot{v}(s)}{\phi(s)}=\frac{s g}{s-Y_{v}}
$$

Frequency response of the $\dot{v}(s) / \phi(s)$ is given in Fig. 7a. As shown in the figure, coherence value is almost 1 which indicates the linearity of the response. $\dot{v}(s) / \phi(s)$ transfer function is fitted on the frequency response between 1-5 rad/s by using NAVFIT module in the CIFER. The cost value of this fitting process is calculated as 1.034. As a result, Eq. (11) is obtained which indicates that $Y_{v}=-0.26068$.

$$
\frac{\dot{v}}{\phi}=\frac{9.806 s}{s+0.26068}
$$

In a similar way with the lateral specific force expression, longitudinal specific force can be defined as shown in Eq. (12).

$$
\dot{u}=X_{u} u-g \theta
$$

After calculating the time derivative and performing the Laplace transform, transfer function of $\dot{u}(s) / \theta(s)$ is generated as shown in Eq. (13)..

$$
\frac{\dot{u}(s)}{\theta(s)}=\frac{-s g}{s-X_{u}}
$$



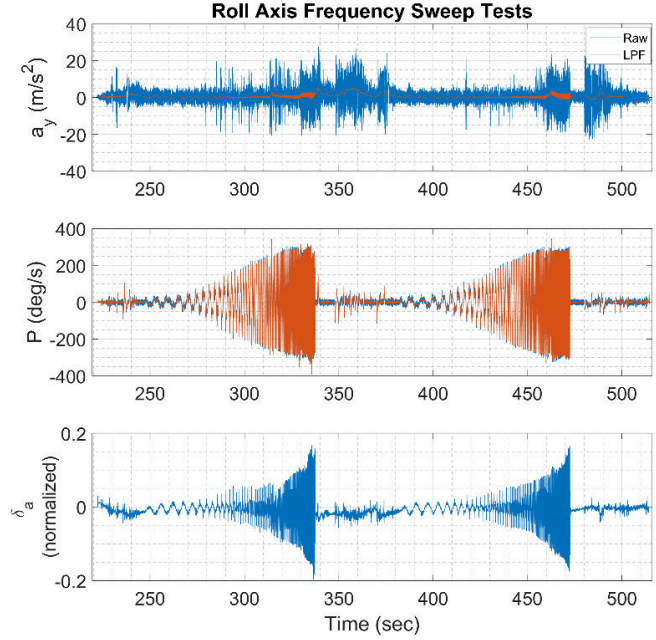

(a)
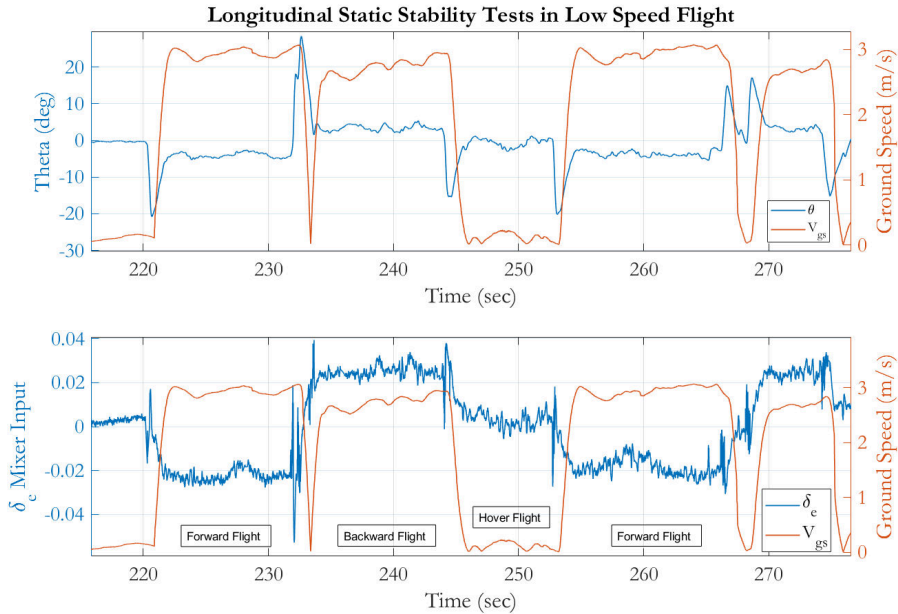

(b)

Fig. 6 a) Roll axis frequency sweep tests. b) LSS flight test in near hover flight conditions.

Frequency response of the $\dot{u}(s) / \theta(s)$ is given in Fig. 7a. As shown in the figure, coherence value is almost 1 between $1-30 \mathrm{rad} / \mathrm{s}$ frequency range which indicates the linearity of the response.

Transfer function fitting process is applied for $1-5 \mathrm{rad} / \mathrm{s}$ frequency range. The obtained transfer function is given in Eq. (14) with the fitting cost value of 3.880. According to this transfer function, longitudinal speed damping derivative is calculated as $X_{u}=-0.270617$.

$$
\frac{\dot{u}}{\theta}=\frac{-9.806 s}{s+0.270617}
$$

Estimation of Longitudinal Speed Stability Derivative $M_{u}$

Longitudinal static stability (LSS) tests are performed to calculate the speed stability derivative $M_{u}$ which is dominant in hover and low speed forward flight phases. In trim flight conditions, the pitching angular acceleration should be zero as shown in Eq. (15).

$$
\dot{q}=0=M_{u} \Delta u+M_{w} \Delta w+M_{\delta_{e}} \Delta \delta_{e}
$$

When the equation is solved for $M_{u}$, Eq. (16) is obtained.

$$
M_{u}=-M_{\delta_{e}} \frac{\Delta \delta_{e}}{\Delta u}+M_{w} \frac{Z_{u}}{Z_{w}}
$$

Due to the dominant effects of the first part of the equation, the second part can be neglected. So, to calculate the longitudinal speed stability derivative in the near hover flight conditions, it is required to obtain $\Delta \delta_{e} / \Delta u$ relationship from the low-speed LSS flight tests.

In these tests, legacy velocity tracking control system in ArduCopter is activated and $3 \mathrm{~m} / \mathrm{s}$ constant ground speed reference signal is applied to the closed-loop system. It is important to perform the low-speed LSS flight tests in almost $0 \mathrm{~m} / \mathrm{s}$ wind conditions to observe the input and vehicle response in the low speed regime. A sample time history of the LSS flight test is given in Fig. $6 \mathrm{~b}$.

After performing the LSS flight tests, pitch axis mixer input / ground velocity $\left(\Delta \delta_{e} / \Delta V_{g s}\right)$ ratio is calculated as -0.007467 . Here, it is possible to use the ground velocity $\left(V_{g s}\right)$ instead of the $X_{b}$ axis velocity $u$ in hover/low speed flight conditions because of the small pitch angle and neglected wind. By using this relationship, $M_{u}$ is calculated as shown in Eq. (17).

$$
M_{u}=-M_{\delta_{e}} \frac{\Delta \delta_{e}}{\Delta u}=(-766.7)(-0.007467)=5.72495
$$


Table 1 Identification costs for each input-output pairs.

\begin{tabular}{ccc}
\hline Response & $J_{h v r}$ & $J_{f f}$ \\
\hline$p / \delta_{a}$ & 23.453 & 69.997 \\
$r / \delta_{a}$ & 48.816 & - \\
$a_{y} / \delta_{a}$ & 31.992 & 48.599 \\
$\dot{v} / \delta_{a}$ & 71.426 & 68.978 \\
$\dot{u} / \delta_{e}$ & 118.755 & 141.831 \\
$q / \delta_{e}$ & 126.780 & 212.850 \\
$a_{x} / \delta_{e}$ & 53.733 & 34.916 \\
$r / \delta_{r}$ & 4.2752 & 50.852 \\
$\dot{v} / \delta_{r}$ & - & 90.682 \\
$a_{z} / \delta_{t}$ & 23.476 & - \\
$\dot{w} / \delta_{t}$ & 43.763 & - \\
\hline$J_{\text {ave }_{\delta_{a}}}$ & 43.922 & 62.525 \\
$J_{\text {ave }_{\delta_{e}}}$ & 99.756 & 129.866 \\
$J_{\text {ave }_{\delta_{r}}}$ & 4.2752 & 70.767 \\
$J_{\text {ave }_{\delta_{t}}}$ & 33.619 & - \\
\hline & &
\end{tabular}

\section{System Identification Results for Hover and Forward Flight Conditions}

The technology demonstrator quadrotor platform has a wide flight envelope covers hover and forward flight conditions with $32 \mathrm{~m} / \mathrm{s}$ maximum total airspeed. Hence, it is important to evaluate the MAV dynamics in these phases. However, because of transmitter range constraints, linear models are generated for hover and $20 \mathrm{~m} / \mathrm{s}$ forward flight conditions. After performing several frequency sweep tests in hover and fast forward flight conditions, recorded data is processed to obtain the frequency responses of the open-loop quadrotor platform.

Measured acceleration and body rate data are used to obtain the non-parametric model, i.e. frequency responses, of the quadrotor roll, pitch, yaw and heave dynamics. Then, linear parametric state-space model is fitted on the obtained frequency responses of the actual system by using the DERIVID tool of the CIFER. The frequency responses of the identified and actual bare-airframe dynamics in hover and forward flight conditions are compared in Fig. 18, 19 and 20 in Appendix. Here, solid and dashed lines represent the frequency responses of the actual system and identified parametric mathematical model, respectively. As shown in these figures, coherence of the frequency responses are above 0.6 for a wide range of frequency which indicates the linearity of the dynamics.

Identified model costs for lateral, longitudinal, directional and heave axis are given in Table 1. Subscripts ' $h v r^{\prime}$ and ' $f f^{\prime}$ are used to indicate hover and forward flight phases. Here, it is shown that individual $\left(J_{i}\right)$ and average $\left(J_{\text {ave }}\right) \operatorname{cost}$ values for the identification process are within or near the suggested limits given in Eq. (18) [13].

$$
\begin{aligned}
& J_{i} \leq 150 \text { to } 200 \\
& J_{\text {ave }} \leq 100
\end{aligned}
$$

Identified aerodynamic parameters of the quadrotor bare-airframe dynamics for hover and forward flight phases are given in Table 2. Several parameters, which have high Cramer-Rao bound $(\geq 25 \%)$ and insensitivity $(\geq 10 \%)$, are neglected and set to zero in the model reduction step. These are marked with $\left(^{+}\right)$superscript in the table.

As shown in Table 2, speed damping and stability derivatives $X_{u}, Y_{v}$ and $M_{u}$ are identified with low Cramer-Rao bound and insensitivities. These results are also verified by the given speed stability and damping analysis in Section IV.B.

\section{Identification of the Propulsion System Dynamics}

Because of the high natural frequency of the BLDC motors, propulsion dynamics may not be captured in the low frequency range. So, it is required to perform a high frequency sweep test to identify the propulsion system dynamics. 
Table 2 Identified parameters for hover and forward flight conditions.

\begin{tabular}{|c|c|c|c|c|c|c|c|c|c|}
\hline & \multirow[b]{2}{*}{ Derivative } & \multicolumn{4}{|c|}{$\mathrm{Vs}=0 \mathrm{~m} / \mathrm{s}$} & \multicolumn{4}{|c|}{$\mathrm{Vs}=20 \mathrm{~m} / \mathrm{s}$} \\
\hline \multirow{8}{*}{$\begin{array}{l}\text { Longitudinal } \\
\text { Model }\end{array}$} & & Value & CR Bound & CR Bound (\%) & Insensitivity (\%) & Value & CR Bound & CR Bound (\%) & Insensitivity (\%) \\
\hline & $X_{u}$ & -0.25860 & 0.01812 & 7.007 & 2.274 & -0.1635 & 0.01764 & 10.79 & 4.698 \\
\hline & $X_{q}$ & -0.07132 & $9.992 \mathrm{E}-03$ & 14.01 & 5.033 & -0.2107 & 0.01649 & 7.828 & 3.201 \\
\hline & $X_{\delta_{e}}$ & -9.124 & 0.4095 & 4.488 & 1.6 & $0^{+}$ & - & - & - \\
\hline & $M_{u}$ & 5.688 & 0.3699 & 6.503 & 1.724 & 3.571 & 0.2903 & 8.128 & 2.338 \\
\hline & $M_{q}$ & 1.958 & 0.3935 & 20.09 & 5.825 & $0^{+}$ & - & - & - \\
\hline & $M_{\delta_{e}}$ & 765.7 & 26.51 & 3.462 & 0.9258 & 753.0 & 36.58 & 4.858 & 1.242 \\
\hline & $\tau_{\delta_{e}}$ & 0.03368 & $2.680 \mathrm{E}-03$ & 7.958 & 3.218 & 0.05124 & $2.292 \mathrm{E}-03$ & 4.474 & 2.003 \\
\hline \multirow{13}{*}{$\begin{array}{l}\text { Lateral } \\
\text { Model }\end{array}$} & $Y_{v}$ & -0.2615 & 0.01644 & 6.289 & 2.446 & -0.3413 & 0.01906 & 5.584 & 1.656 \\
\hline & $Y_{p}$ & $0^{+}$ & - & - & - & $0^{+}$ & - & - & - \\
\hline & $Y_{r}$ & $0^{+}$ & - & - & - & $0^{+}$ & - & - & - \\
\hline & $L_{v}$ & -6.154 & 0.3272 & 5.317 & 1.783 & -0.7775 & 0.05845 & 7.518 & 2.233 \\
\hline & $L_{p}$ & $0^{+}$ & - & - & - & -1.532 & 0.2521 & 16.45 & 6.770 \\
\hline & $L_{r}$ & $0^{+}$ & - & - & - & $0^{+}$ & - & - & - \\
\hline & $N_{v}$ & 1.169 & 0.3145 & 26.91 & 6.279 & $0^{+}$ & - & - & - \\
\hline & $N_{p}$ & 0.2032 & 0.03175 & 15.62 & 3.464 & $0^{+}$ & - & - & - \\
\hline & $N_{r}$ & $0^{+}$ & - & - & - & $0^{+}$ & - & - & - \\
\hline & $Y_{\delta_{a}}$ & 12.07 & 0.5377 & 4.456 & 1.747 & 7.036 & 1.365 & 19.41 & 6.580 \\
\hline & $L_{\delta_{a}}$ & 1232 & 38.12 & 3.094 & 0.9929 & 913.4 & 30.57 & 3.347 & 1.045 \\
\hline & $N_{\delta_{a}}$ & 18.50 & 1.361 & 7.357 & 3.545 & $0^{+}$ & - & - & - \\
\hline & $\tau_{\delta_{a}}$ & 0.03323 & $2.205 \mathrm{E}-03$ & 6.637 & 2.976 & 0.03898 & $1.360 \mathrm{E}-03$ & 3.489 & 1.651 \\
\hline \multirow{9}{*}{$\begin{array}{c}\text { Directional } \\
\text { Model }\end{array}$} & $N_{r}$ & $0^{+}$ & - & - & - & -21.44 & 3.234 & 15.08 & 0.2437 \\
\hline & $Y_{v}$ & $0^{+}$ & - & - & - & 15.11 & 3.107 & 20.56 & 0.2740 \\
\hline & $Y_{r}$ & $0^{+}$ & - & - & - & 9.188 & 1.249 & 13.59 & 0.1818 \\
\hline & $N_{\delta_{r}}$ & 92.71 & 8.614 & 9.284 & 4.028 & 122.0 & 9.521 & 7.807 & 0.3676 \\
\hline & $Y_{\delta_{r}}$ & $0^{+}$ & - & - & - & 35.67 & 4.724 & 13.24 & 0.4037 \\
\hline & $N_{v}$ & $0^{+}$ & - & - & - & 51.02 & 9.108 & 17.85 & 0.2593 \\
\hline & $\omega_{\delta_{r}}$ & -26.04 & 4.708 & 18.06 & 3.468 & $-26^{+}$ & - & - & - \\
\hline & $\tau_{\text {lead }}$ & -16.13 & 1.608 & 9.978 & 2.193 & -2.313 & 0.2286 & 9.883 & 2.807 \\
\hline & $\tau_{\delta_{r}}$ & 0.01891 & $2.253 \mathrm{E}-03$ & 11.91 & 4.309 & $0^{+}$ & - & - & - \\
\hline \multirow{4}{*}{$\begin{array}{l}\text { Heave } \\
\text { Model }\end{array}$} & $Z_{w}$ & $0^{+}$ & - & - & - & & & & \\
\hline & $Z_{\delta_{t}}$ & -70.27 & 2.808 & 3.996 & 1.501 & - & - & - & - \\
\hline & $\omega_{\delta_{t}}$ & -25.6 & 2.307 & 9.012 & 2.923 & - & - & - & - \\
\hline & $\tau_{\delta_{t}}$ & $6.514 \mathrm{E}-03$ & $1.730 \mathrm{E}-03$ & 26.57 & 10.49 & - & - & - & - \\
\hline
\end{tabular}

For this purpose, the frequency sweep flight tests are repeated for the pitch and roll axes.

If we ignore the low-frequency hovering cubics, pitch and roll dynamics of the quadrotor bare-airframe can be modelled as second-order transfer functions in the high frequency region. So, linearized high frequency dynamics are represented as shown in Eq. (19) and (20) which combine the frame and BLDC motor dynamics.

$$
\begin{gathered}
\frac{p(s)}{\delta_{a}(s)}=\frac{L_{\delta_{a}}\left(\omega_{\phi}\right) e^{-\tau_{\phi} s}}{s\left(s+\omega_{\phi}\right)} \\
\frac{q(s)}{\delta_{e}(s)}=\frac{M_{\delta_{e}}\left(\omega_{\theta}\right) e^{-\tau_{\theta} s}}{s\left(s+\omega_{\theta}\right)}
\end{gathered}
$$

where, $\omega_{\theta}$ and $\omega_{\phi}$ are natural frequencies of the propulsion system, $\tau_{\theta}$ and $\tau_{\phi}$ are high frequency delays on roll and pitch axes. Then, transfer functions are fitted on the identified $p(s) / \delta_{a}(s)$ and $q(s) / \delta_{e}(s)$ frequency responses as shown in Fig. 7b.

The identified linear models for the high frequency range are given in Eq. (21) and (22) with the cost of 3.992 and 1.009 , respectively. As shown in these identified transfer functions, natural frequency of the propulsion system is obtained as approximately $29 \mathrm{rad} / \mathrm{s}$. 


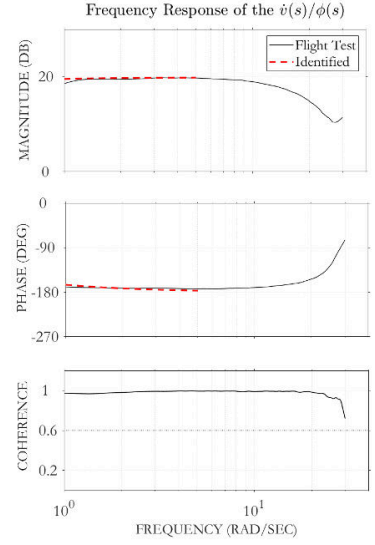

(a)
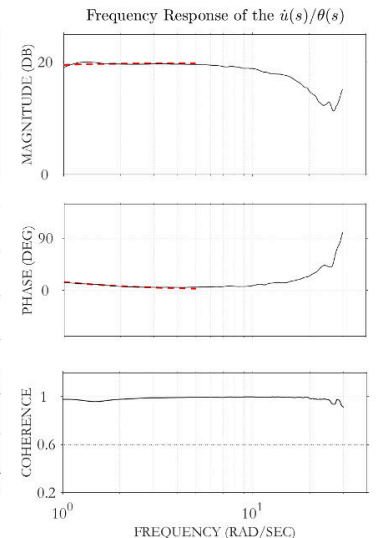
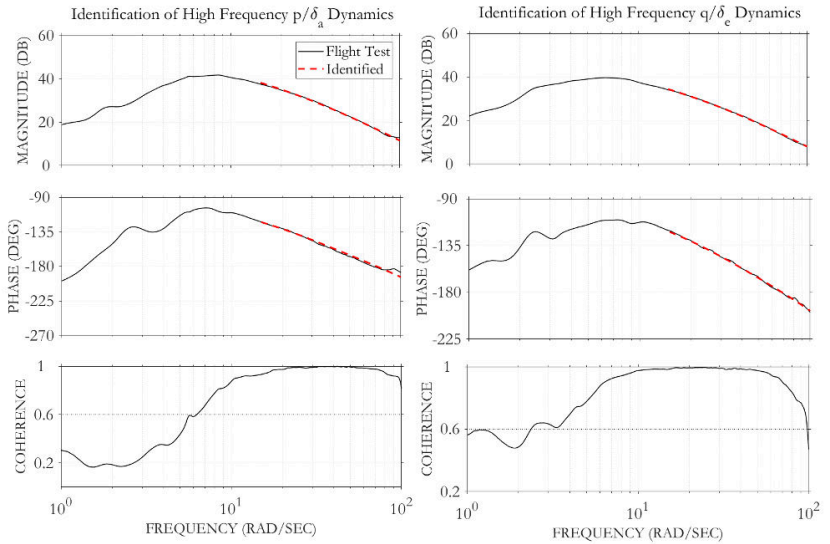

(b)

Fig. 7 a) Frequency responses of the $\dot{v}(s) / \phi(s)$ and $\dot{u}(s) / \theta(s)$. b) Identification of high frequency dynamics on longitudinal and lateral axes.

$$
\begin{aligned}
& \frac{p(s)}{\delta_{a}(s)}=\frac{L_{\delta_{a}}\left(\omega_{\phi}\right) e^{-\tau_{\phi} s}}{s\left(s+\omega_{\phi}\right)}=\frac{39018.6 e^{-0.0052 s}}{s(s+28.276 s)} \\
& \frac{q(s)}{\delta_{e}(s)}=\frac{M_{\delta_{e}}\left(\omega_{\theta}\right) e^{-\tau_{\theta} s}}{s\left(s+\omega_{\theta}\right)}=\frac{26229.3 e^{-0.0060 s}}{s(s+29.695 s)}
\end{aligned}
$$

\section{E. Model Verification}

Identified linear models of the quadrotor platform should be verified before using in the control system design process. For this purpose, verification test procedure is utilized in the time-domain. In these tests, doublet reference attitude signals with $\simeq \pm 10 \%$ amplitude of the pilot stick range are applied on each axis, separately. Then, mixer input and system output data are logged on the SD card. Mixer inputs are applied into the identified linear bare-airframe models and responses are compared with the actual system outputs.

Model verification flight tests are performed by using stabilize mode of the ArduCopter. In this mode, pilot sends attitude commands by using the RC transmitter. Then, verification test data is processed by using VERIFY tool in CIFER. Because of the unstable bare-airframe dynamics, linear model is simulated for about 1.5-2 seconds to avoid the unstable model responses. Roll axis verification results are given in Fig. 8. As shown in this figure, actual system and linear model have similar responses which verifies the system identification results.

In the model verification process, Theil-inequality constant (TIC) and verification cost $\left(J_{v r f}\right)$ values are used to evaluate the similarity of the identified model and actual system responses. According to the guideline in [13], TIC and $J_{v r f}$ values should be as given in Eq. (23) and (24).

$$
\begin{gathered}
T I C \leq 0.25 \text { to } 0.30 \\
J_{v r f} \leq 1.0 \text { to } 2.0
\end{gathered}
$$

Model verification results for hover and forward flight conditions are given in Table 4. As shown in this table, TIC and $J_{v r f}$ values are below the given limits in Eq. (23) and (24). Here, subscripts ' $h v r^{\prime}$ and ' $f f^{\prime}$ are used for hover and forward flight phases, respectively.

In the next section, model stitching method is introduced which is used to generate a full-flight envelope simulation environment by using linear point models and trim dataset. 


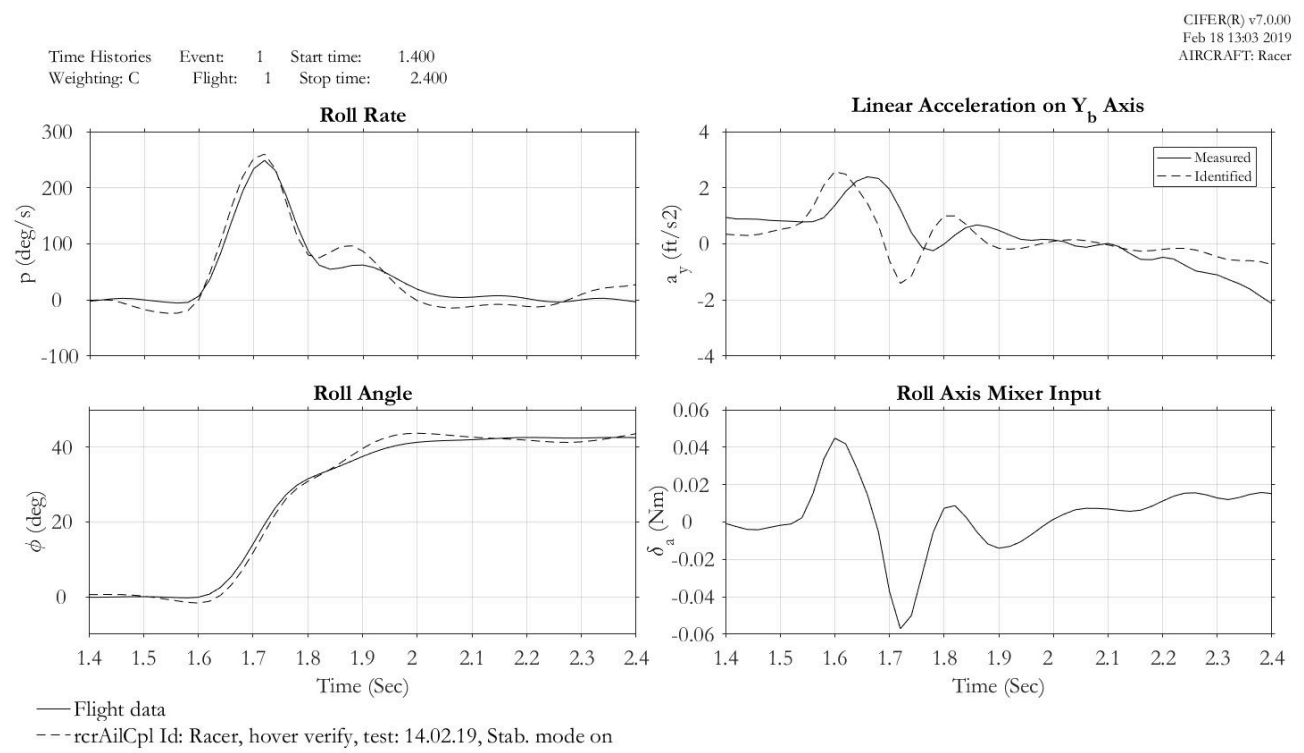

Fig. 8 Roll axis verification test results in hover flight.

Table 3 Test signal parameters for propulsion system identification in high frequency range.

\begin{tabular}{ll}
\hline Parameter & Value \\
\hline Frequency Range & $1-100 \mathrm{rad} / \mathrm{s}$ \\
Sweep Length & $90 \mathrm{sec}$ \\
Sampling Time & $400 \mathrm{~Hz}$ \\
Concatenated Record Length & $200 \mathrm{sec}$ \\
\hline
\end{tabular}

Table 4 Verification TIC and cost $\left(J_{v r f}\right)$ values for hover and forward flight conditions.

\begin{tabular}{ccccc}
\hline Axis & $T I C_{h v r}$ & $J_{v r f_{h v r}}$ & $T I C_{f f}$ & $J_{v r f_{f f}}$ \\
\hline Roll & 0.06834 & 1.804 & 0.05307 & 0.723 \\
Pitch & 0.09661 & 1.632 & 0.04378 & 1.251 \\
Yaw & 0.06953 & 1.914 & 0.03334 & 0.760 \\
\hline
\end{tabular}

\section{Model Stitching}

Linear state-space perturbation models represent the dynamical behaviour of the aerial system and critical dynamics can be captured by using these simple mathematical models. However, linear models are valid only for a specific flight condition. If the aerial vehicle has a wide flight envelope, one linear model would be insufficient to capture the dynamical behaviour in the whole envelope. Hence, linear models should be obtained for several flight conditions and control system parameters should be scheduled based on airspeed, dynamic pressure or altitude.

As we mentioned in the previous sections, the system identification process is a powerful tool to obtain the linear mathematical model of the aerial vehicle for a specific flight condition. However, it is required to generate a full-flight envelope simulation environment to evaluate the closed-loop system behaviour. For this purpose, model stitching method is developed in which several anchor points and trim data are stitched together to cover the flight envelope of the aerial vehicle $[11,13,15]$. General structure of the stitched model is given in Fig. 9.

\section{A. Anchor Point Data}

Anchor point data is defined as a specific flight condition in which the linear model and trim data are available. For the agile maneuvering quadrotor platform, two anchor point models (for hover and $20 \mathrm{~m} / \mathrm{s}$ forward flight) are identified and used in the stitched model. Stability and control derivatives are linearly interpolated between these two point models as a function of $U$. To obtain the anchor trim point, forward flight tests are performed in several pitch attitude between $0^{\circ}$ and $-55^{\circ}$. Then, $W_{0}, \Theta_{0}, \delta_{e_{0}}$ and $\delta_{t_{0}}$ are calculated by using the trim flight data records as shown in Fig. 10. This data set is used to obtain the stitched model which covers the hover/low speed and fast forward flight phases. For more information and comprehensive analysis about the model stitching method, readers may refer to [11, 13, 15].

In the next section, trajectory tracking control system structure is given, controller parameters are optimized by using multi-objective optimization process and robustness analysis is performed. 


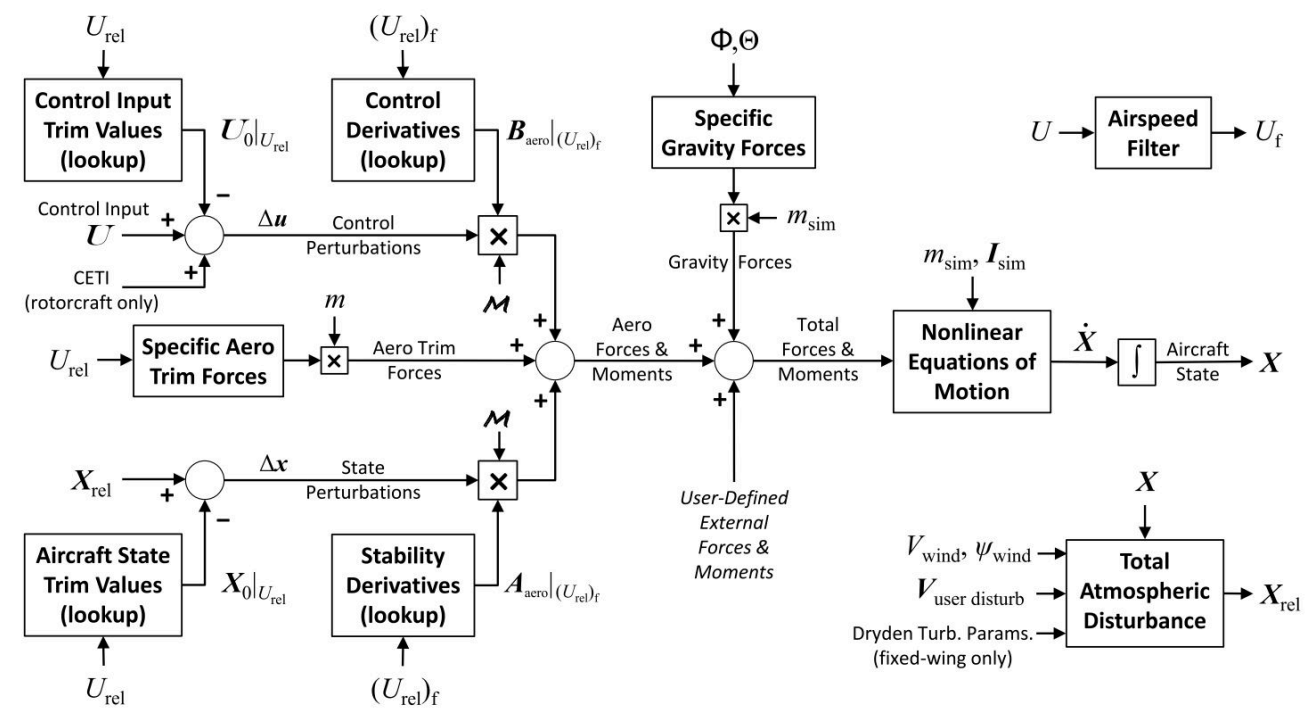

Fig. 9 General view of the stitch model structure [15].
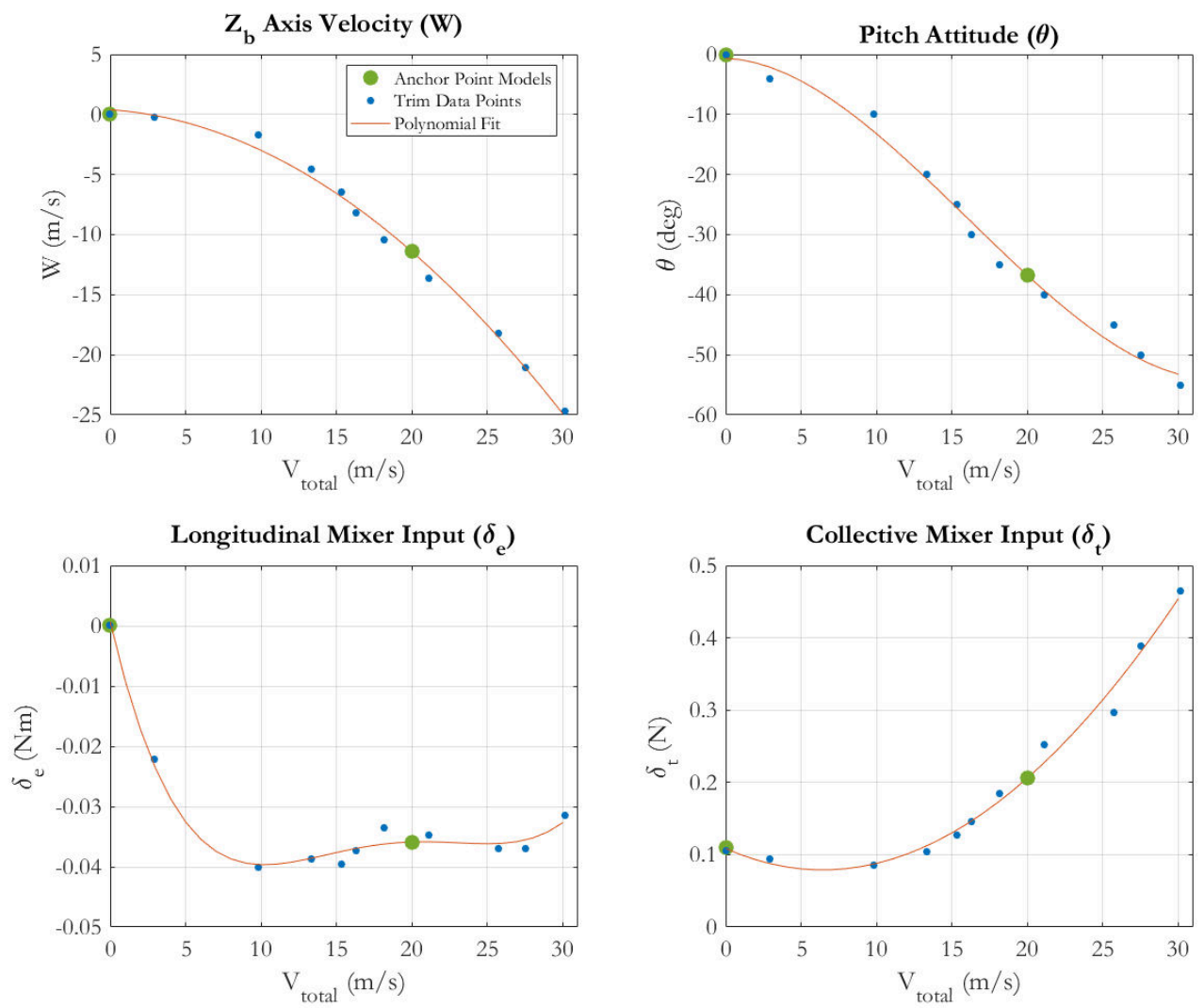

Fig. 10 Anchor point models and trim data obtained from the forward flight tests in trim conditions. 


\section{Control System Design}

Legacy ArduCopter control system has a nested-loop structure which contains attitude, velocity and position control loops. By using this legacy structure and controller parameters, it is possible to perform general purpose flights in which it is not critical to track a given reference signal precisely. However, it is not suitable for accurate trajectory tracking applications such as B-spline tracking missions. For this reason, the legacy structure is modified and controller parameters are optimized for the agile maneuvering quadrotor platform. At first, stability and performance of the legacy attitude controllers are evaluated by using the frequency domain analysis. These results are used to compare the legacy and optimized controller performances quantitatively. Then, inner and outer-loop controllers are optimized in Control Designer's Unified Interface (CONDUIT) software [1] by using selected handling qualities.

\section{A. ArduCopter Controller Structures}

ArduCopter controllers are used as legacy control systems which is developed based on nested-loop structure [16]. The innermost loop of the controller structure contains a PID controller which is utilized to control the body rates of the quadrotor. Output of the inner-loop rate controller is sent to the mixer to obtain the required PWM signal for each BLDC motor. A proportional controller is used in the second loop to track the given attitude references. Also, a feed-forward element is used in this loop. The third loop of the ArduCopter controller is in PID structure and designed to track given velocity reference signals in the NED frame. The outermost loop is designed based on a proportional control structure and utilized to track the given position reference. Block diagram of the ArduCopter controller is shown in Fig. 11.

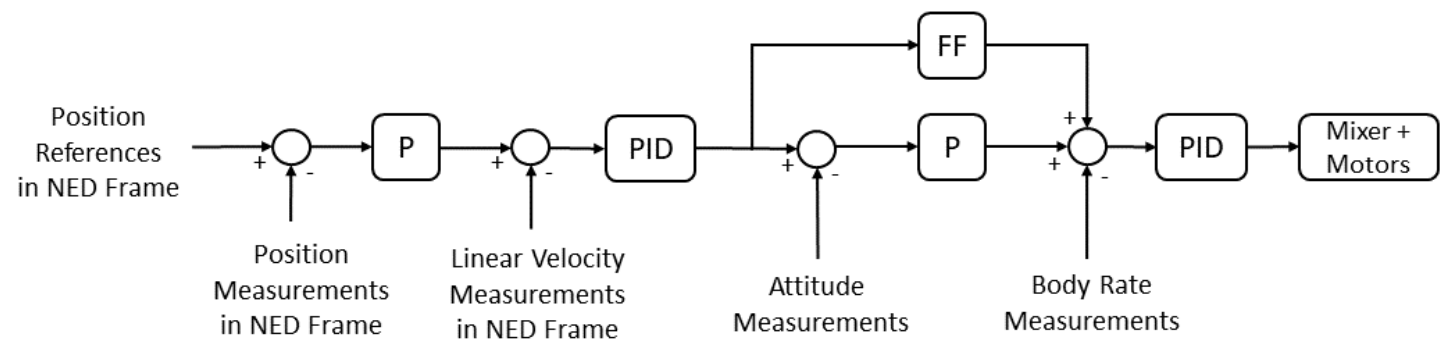

Fig. 11 Block diagram of the ArduCopter Position control system.

As shown in Fig.11, only the position reference signal can be applied into the legacy closed-loop system. It is not possible to track the given position and velocity references simultaneously. Hence, the position and velocity control loops of the legacy structure have to be modified. To simplify the overall design process, attitude control loop structure is kept same. However, it is important to evaluate the stability and performance of the legacy attitude controller as given in the following section.

\section{B. ArduCopter Inner-loop Attitude Controller Performance Evaluation}

Before improving the legacy controller performance or designing a new controller structure, it is important to obtain the broken-loop (BL), closed-loop (CL) and disturbance rejection (DR) performance of the baseline inner-loop attitude control system. This information provides a prior knowledge about the required improvement that should be performed by the control engineer. Hence, to determine the dynamical characteristics of the ArduCopter, broken-loop, closed-loop and disturbance rejection flight tests are performed by using the legacy controller. The input-output pairs for these tests are given in Fig. 12 where, subscripts ' $r e f^{\prime},{ }^{\prime} b l^{\prime}$ and ' $d$ ' refer to the reference, broken-loop and disturbance inputs, respectively. Frequency sweep flight test is designed to obtain the frequency response of BL, CL and DR dynamics. Sweep signal parameters are given in Table 5.

To evaluate the stability margins and crossover frequencies of the legacy attitude controllers, the broken-loop flight

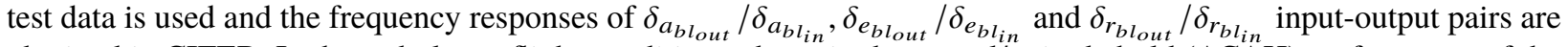
obtained in CIFER. In the turbulence flight conditions, the attitude control/attitude hold (ACAH) performance of the UAV has a crucial role to track the given reference signal. Hence, disturbance rejection bandwidth and disturbance rejection peak characteristics are analyzed by using the frequency responses of the $\phi^{\prime} / \phi_{d}, \theta^{\prime} / \theta_{d}$ and $\psi^{\prime} / \psi_{d}$ input-output pairs in the CIFER software. Another important reference signal tracking performance metric is the bandwidth of the closed-loop system. It is required to design an inner-loop system with adequate bandwidth to track given reference signal by the outer-loop, such as trajectory tracking system. Therefore, the closed-loop system performance of the 


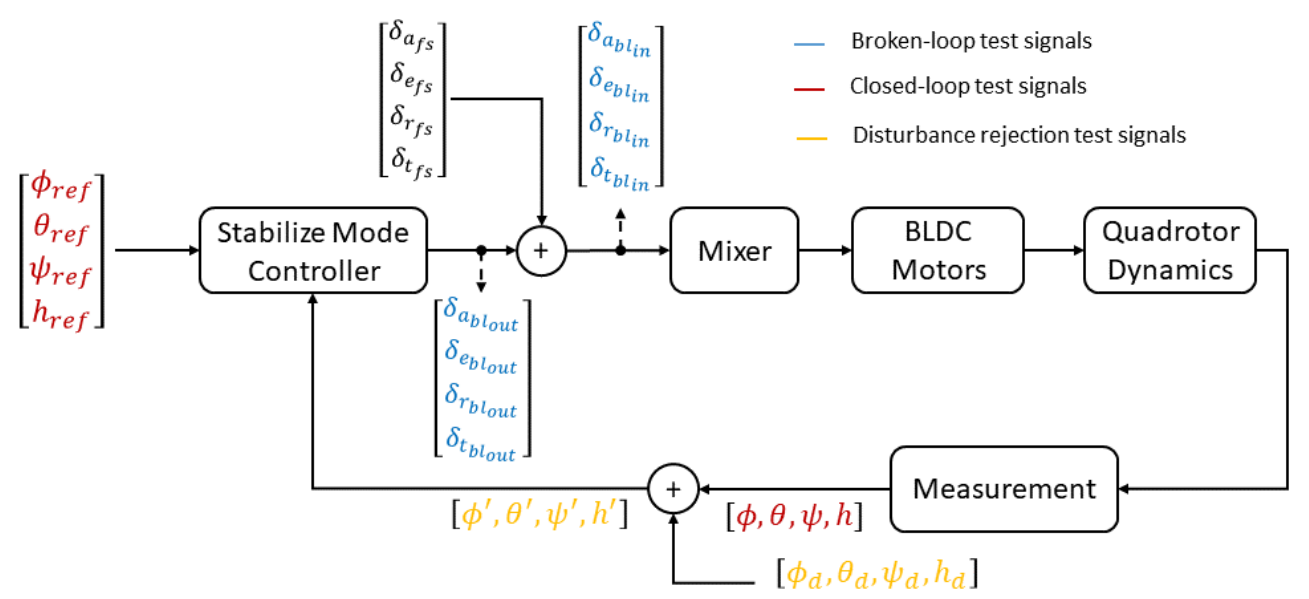

Fig. 12 Schematic of the closed-loop, broken-loop and disturbance rejection test input-output pairs.

Table 5 Frequency sweep signal parameters for Arducopter performance evaluation tests.

\begin{tabular}{cccc}
\hline Parameter & Broken-loop & Closed-loop & Disturbance Rejection \\
\hline$T_{\text {sweep }}$ & $90 \mathrm{sec}$ & $90 \mathrm{sec}$ & $90 \mathrm{sec}$ \\
$\omega_{\min }$ & $1 \mathrm{rad} / \mathrm{s}$ & $1 \mathrm{rad} / \mathrm{s}$ & $1 \mathrm{rad} / \mathrm{s}$ \\
$\omega_{\max }$ & $100 \mathrm{rad} / \mathrm{s}$ & $90 \mathrm{rad} / \mathrm{s}$ & $90 \mathrm{rad} / \mathrm{s}$ \\
$T_{s}$ & $400 \mathrm{~Hz}$ & $400 \mathrm{~Hz}$ & $400 \mathrm{~Hz}$ \\
Pitch Axis Test Signal Amp. & $0.05 \mathrm{Nm}$ & $\pm 10 \mathrm{deg}$ & $\pm 15 \mathrm{deg}$ \\
Roll Axis Test Signal Amp. & $0.05 \mathrm{Nm}$ & $\pm 10 \mathrm{deg}$ & $\pm 15 \mathrm{deg}$ \\
Yaw Axis Test Signal Amp. & $0.1 \mathrm{Nm}$ & $\pm 20 \mathrm{deg} / \mathrm{s}$ & $\pm 20 \mathrm{deg} / \mathrm{s}$ \\
\hline
\end{tabular}

legacy attitude controller is evaluated by using the frequency responses of $\phi / \phi_{c}, \theta / \theta_{c}$ and $\psi / \psi_{c}$ input-output pairs in the CIFER.

The frequency-domain stability and performance characteristics of the actual legacy controller is also used to verify the mathematical model of the closed-loop system (controller, sensor dynamics and bare-airframe) in CONDUIT environment. BL, CL and DR flight test results are given in Table 6. Handling quality analysis of the legacy roll attitude controller from CONDUIT is given in Fig. 21 in Appendix.

As shown in the Table 6 and Fig. 21, the legacy controllers have adequate crossover frequency and disturbance rejection bandwidth characteristics. However, they have low phase margin which results in low damping ratio of the closed-loop system. Also, the closed-loop system with legacy controller has Nichols Margin in Level-3 which indicates that the system is not robust against simultaneous changes in magnitude and phase. This situation reduces the stability of the system. In the fast forward flight tests, effect of the low stability margin characteristics of the attitude control loop is observed as low-damping oscillations in pitch and roll attitude of the quadrotor platform. This results insufficient trajectory tracking performance in the fast forward flight phase. Hence, it is required to improve the inner-loop reference tracking performance of the attitude control loop.

\section{Dynamic Scaling}

In literature, there are several sources about handling quality requirements for manned aerial vehicles. For manned rotorcrafts and fixed-wing aircrafts, ADS-33E-PRF and MIL-STD-1797B provide a comprehensive set of handling and flying qualities. However, there is very limited information about the handling quality requirements for UAVs and MAVs. In recent years, researchers have focused on dynamic (Froude) scaling to scale down the Level-1 boundaries of the several handling quality requirements such as disturbance rejection bandwith (DRB) and crossover frequency $\left(\omega_{c}\right)$. Scaling factor is calculated by using the rotor diameter (for full scale rotorcrafts) and the hub-to-hub distance (for 
Table 6 Performance evaluation of the legacy control systems.

\begin{tabular}{|c|c|c|c|c|c|c|c|c|}
\hline Axis & & $\begin{array}{c}\omega_{c} \\
(\mathrm{rad} / \mathrm{s})\end{array}$ & $\begin{array}{c}\text { PM } \\
(\operatorname{deg})\end{array}$ & $\begin{array}{l}\text { GM } \\
(\mathrm{dB})\end{array}$ & $\begin{array}{c}-135 \operatorname{deg} \text { BW } \\
(\mathrm{rad} / \mathrm{s})\end{array}$ & $\begin{array}{c}-180 \operatorname{deg} \mathrm{BW} \\
(\mathrm{rad} / \mathrm{s})\end{array}$ & $\begin{array}{c}\text { DRB } \\
(\mathrm{rad} / \mathrm{s})\end{array}$ & $\begin{array}{l}\text { DRP } \\
(\mathrm{dB})\end{array}$ \\
\hline Roll Attitude & Flight Test & 34.64 & 23 & $13.08 \mathrm{~dB} @ 5.53 \mathrm{rad} / \mathrm{s}$ & 33.41 & 46 & 9.725 & 2.51 \\
\hline Controller & CONDUIT & 29.3 & 33.33 & $19.34 \mathrm{~dB} @ 7.42 \mathrm{rad} / \mathrm{s}$ & 31.95 & 51.74 & 11.96 & 4.87 \\
\hline Pitch Attitude & Flight Test & 20.9 & 33.6 & $9.69 \mathrm{~dB} @ 5.4 \mathrm{rad} / \mathrm{s}$ & 22.7 & 38.20 & 8.217 & 2.9 \\
\hline Controller & CONDUIT & 19.33 & 24.39 & $9.93 \mathrm{~dB} @ 9.49 \mathrm{rad} / \mathrm{s}$ & 21.1 & 37.34 & 10.09 & 7.47 \\
\hline
\end{tabular}

Table 7 Froude scaling analysis of the agile maneuvering drone roll mode in hover flight.

\begin{tabular}{ccccc}
\hline Mode & XV-15 & Scaled XV-15 & Agile Drone & Difference (\%) \\
\hline$\omega_{n_{\text {roll }_{1,2}}}(\mathrm{rad} / \mathrm{s})$ & 0.4668 & 3.8532 & 3.8785 & 0.6556 \\
$\omega_{n_{\text {roll }_{3}}}(\mathrm{ad} / \mathrm{s})$ & 0.6458 & 5.3308 & 4.0114 & 24.7501 \\
\hline
\end{tabular}

multicopter platforms) of the aerial vehicles $[4,17]$.

In this study, XV-15 full-scale tilt-rotor aircraft is used to perform the Froude scaling analysis. Scaling factor $N$ is calculated as the ratio of the rotor-to-rotor distance of the XV-15 $\left(L_{x v 15}\right)$ and the rotor-to-rotor diagonal distance of the technology demonstrator drone platform $\left(L_{t d}\right)$ as shown in Eq. (25).

$$
N=\frac{L_{x v 15}}{L_{t d}}=\frac{17.374}{0.255}=68.137
$$

By using the dynamical scale factor $N$, full-scale frequency requirements can be scaled down by using Eq. (26).

$$
\omega_{\text {scaled }}=\omega_{\text {full-scale }} \sqrt{N}
$$

To evaluate the validity of the dynamical scaling, actual and scaled natural frequencies of the XV-15 roll mode poles are compared with the identified roll mode poles of the drone platform in Table 7. Here, it is shown that the difference between the natural frequencies of the scaled and identified roll axis hovering cubic poles are $0.6562 \%$ and $24.7501 \%$, respectively. This indicates that the Froude scaling can be used to obtain the scaled handling quality requirements for the quadrotor platform.

\section{Trajectory Tracking Control System Design}

A suitable control system structure is necessary to perform the trajectory tracking mission with a minimum error in velocity and position. The legacy ArduCopter position control system has a classical nested-loop structure as mentioned before. By using this structure, it is possible to track the given position reference signal. However, simultaneous tracking of the position and velocity commands, which is required for the trajectory tracking mission, is not possible with the legacy closed-loop system. Hence, a modification on the position control loop is performed and the position controller signal is used as a correction on the velocity reference signal. The general scheme of the trajectory tracking system is given in Fig. 13.

As mentioned in the previous sections, ArduCopter is used as a legacy control system for the Racer quadrotor platform. System identification and model verification tests are performed by using the legacy controller because of the inherent unstable dynamics of the quadrotor. Then, in Section VI.B, broken-loop, closed-loop and disturbance rejection characteristics are evaluated in frequency-domain. As a result of these analysis, it is shown that the legacy attitude controllers have low phase margin. Hence, it may not be possible to perform an accurate and agile trajectory tracking mission by using the legacy controllers. At this point, modifying the controller structure and optimizing its parameters become necessary to improve the reference tracking performance.

In this study, the legacy attitude controller structure (P+PID) is not changed to simplify the problem. CONDUIT is used to optimize the attitude control system parameters by using the selected stability and handling quality specifications given in Table 8. In CONDUIT, flight control system design problem is constrained by these selected specifications 


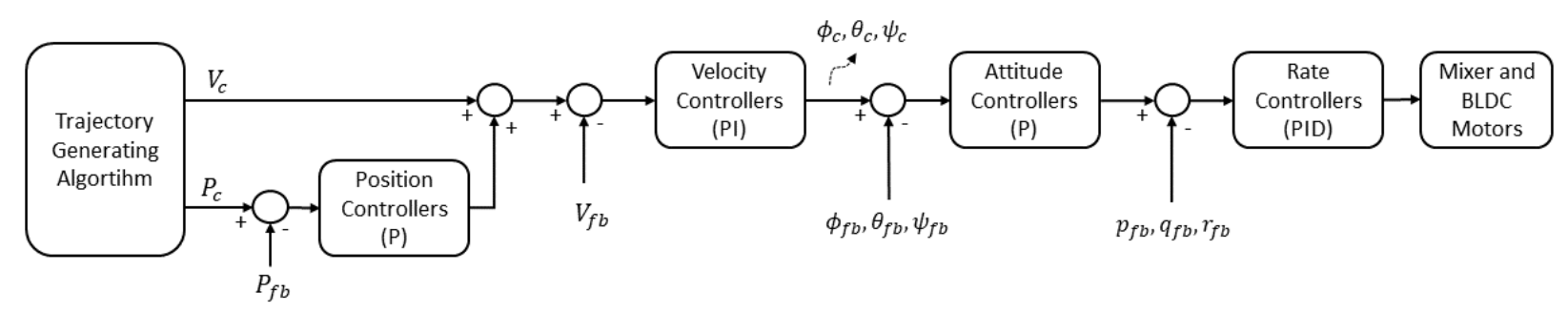

Fig. 13 Block diagram of the proposed trajectory tracking system.

and Feasible Sequential Quadratic Programming (FSQP) solver is used to obtain the pareto-optimum solution with the minimum overdesign [1]. However, most of the design specifications, such as minimum crossover frequency and disturbance rejection bandwidth requirements, are not suitable for unmanned aerial vehicles. In the next two subsections, it is described how to modify the level- 1 boundaries of these specifications according to bare-airframe dynamics and Froude scaling analysis to obtain suitable specifications for UAV and MAV platforms.

\section{Minimum Crossover Frequency}

One of the main characteristics of the feedback systems is its suppression ability against the variations in the system dynamics due to mass, airspeed and center of gravity location, etc. If the parameter variation has significant effects on the dynamical characteristics of the system, consistent response can be obtained by using high feedback gains. This increases the crossover frequency of the broken-loop system at the cost of increased actuator activity. Hence, it is important to select a minimum crossover boundary to avoid the overdesign. According to the guideline in [1], the minimum crossover frequency boundary should be selected at least 2 to 3 times greater than the natural frequency of the unstable modes of the bare-airframe as shown in Eq. (27).

$$
\omega_{c} \geq(2-3) \omega_{\text {unstable }}
$$

In the agile maneuvering quadrotor platform, identified unstable mode natural frequencies of the hovering cubics are $3.8785 \mathrm{rad} / \mathrm{s}$ for roll dynamics and $4.0881 \mathrm{rad} / \mathrm{s}$ for short-period dynamics. So, level-1 boundary of the minimum crossover frequency specification (CrsMnG2) of the attitude control loop is selected as $25 \mathrm{rad} / \mathrm{s}$.

Velocity hold controller is wrapped around the attitude hold control system of the quadrotor platform. It generates the commanded pitch and roll attitude to track the given velocity reference signal. Here, it is important to minimize the dynamical interaction between the inner and outer loop. Only a small decrease in the stability margin of the inner loop is acceptable when the outer-loop is added into the nested-loop structure. For this purpose, crossover frequency of the velocity-hold control system is selected to provide frequency separation between the attitude control loop and velocity hold loop. According to the guidelines, crossover frequency of the outer loop is selected as $1 / 5$ to $1 / 3$ of the inner loop crossover frequency as shown in Eq. (28) [1].

$$
\omega_{c_{v e l}}=\left(\frac{1}{5}-\frac{1}{3}\right) \omega_{c_{a t t}}
$$

In the proposed trajectory tracking structure, position controller is used as a correction loop instead of using as an outer-loop. Hence, bandwidth separation is not applied in the position correction loop and crossover frequency of the position controller is selected as $10 \mathrm{rad} / \mathrm{s}$.

\section{Disturbance Rejection Requirements}

Disturbance rejection requirements consists of disturbance rejection bandwidth (DRB) and disturbance rejection peak (DRP) criteria which are used to evaluate the rejection capabilities of the aerial vehicle in a disturbed situation $[1,18]$. In CONDUIT, DRB and DRP specifications are defined as DstBwG1 and DstPkG1, respectively. Although DRP specification can be used for the sub-scale aerial vehicle, DRB specification should be scaled-down by using the Froude scaling in Eq. (25). As a result, level-1 boundary of the attitude DRB specification is shifted to the $8.25 \mathrm{rad} / \mathrm{s}$. Summary of the selected design specifications and Level-1 boundaries are given in Table 8. Here, $H, S, J$ are used to define hard, soft constraints and summed objectives in the optimization problem, respectively. 
Table 8 Summary of the selected design specifications in control system design process.

\begin{tabular}{lccccc}
\hline Specifications & $\begin{array}{c}\text { Nominal Level-1 } \\
\text { Boundary }\end{array}$ & $\begin{array}{c}\text { Roll and Pitch } \\
\text { Attitude Controllers }\end{array}$ & $\begin{array}{c}\text { Yaw Attitude } \\
\text { Controller }\end{array}$ & $\begin{array}{c}\text { Velocity } \\
\text { Controller }\end{array}$ & $\begin{array}{c}\text { Position } \\
\text { Controller }\end{array}$ \\
\hline EigLcG1 $(\mathrm{H})$ & 0 & Unmodified & Unmodified & Unmodified & Unmodified \\
NcMgG1 $(\mathrm{H})$ & - & Unmodified & Unmodified & Unmodified & Unmodified \\
StbMgG1 $(\mathrm{H})$ & $45 \mathrm{deg} / 6 \mathrm{~dB}$ & Unmodified & Unmodified & Unmodified & Unmodified \\
DstBwG1 $(\mathrm{S})$ & $1 \mathrm{rad} / \mathrm{s}$ & $8.25 \mathrm{rad} / \mathrm{s}$ & $2 \mathrm{rad} / \mathrm{s}$ & $1.61 \mathrm{rad} / \mathrm{s}$ & $1.51 \mathrm{rad} / \mathrm{s}$ \\
DstPkG1 $(\mathrm{S})$ & $4.5 \mathrm{~dB}$ & Unmodified & Unmodified & Unmodified & Unmodified \\
EigDpG1 $(\mathrm{S})$ & 0.35 & Unmodified & 0.5 & Unmodified & Unmodified \\
CrsMnG2 $(\mathrm{S})$ & $2 \mathrm{rad} / \mathrm{s}$ & $25 \mathrm{rad} / \mathrm{s}$ & $5 \mathrm{rad} / \mathrm{s}$ & $5 \mathrm{rad} / \mathrm{s}$ & $2.5 \mathrm{rad} / \mathrm{s}$ \\
CrsLnG1 $(\mathrm{J})$ & $10 \mathrm{rad} / \mathrm{s}$ & $40 \mathrm{rad} / \mathrm{s}$ & $10 \mathrm{rad} / \mathrm{s}$ & $10 \mathrm{rad} / \mathrm{s}$ & $10 \mathrm{rad} / \mathrm{s}$ \\
RmsAcG1 $(\mathrm{J})$ & 1.5 & Unmodified & Unmodified & Unmodified & Unmodified \\
\hline
\end{tabular}

\section{E. Robustness Analysis of the Attitude Controllers}

Robustness analysis is a crucial step for the control system design process to evaluate the system behaviour in the presence of parametric uncertainties. So, it is important to calculate the uncertainty level of the system parameters. Each of the aerodynamic parameter identified in the system identification process has a Cramer-Rao (CR) bound which represents the uncertainty level of the related parameter as given in Eq. (29) [13].

$$
\left(C R_{i}\right)_{\text {cifer }} \approx \sigma_{i}
$$

where, $\sigma_{i}$ is the standard deviation of the identified aerodynamic parameter of the aerial vehicle. This uncertainty value is used to evaluate the closed-loop system robustness against the parametric uncertainties in the mathematical model. $\pm 2 C R \approx 2 \sigma_{i}$ and $\pm 3 C R \approx 3 \sigma_{i}$ uncertainty levels in the state-space parameters account for $95.4 \%$ and $99.7 \%$ confidence interval in the mathematical model [13].

In CONDUIT, CR bounds of the identified parameters are imported from the CIFER and 100-runs Monte-Carlo simulation is performed for $3 \sigma_{i}$ uncertainty level. Design margin in DRB and minimum crossover specifications are set as 30\% to keep the closed-loop system in the Level-1 region in the presence of uncertainties. As a result of the robustness analysis, it is shown that the optimized attitude control systems have adequate robustness capability against $3 \sigma_{i}$ parametric uncertainties. Robustness analysis results of the roll axis attitude controller is given in Fig. 22 in Appendix. As shown in Fig. 22, most of the design point remain in level-1 region in the presence of parametric uncertainties. In the worst cases, some of them shift slightly into the level-2 which is still acceptable. Similar results are obtained for the pitch and yaw attitude controllers. However, they are not given here because of the page limitation.

In the next section, closed-loop system performance is evaluated in hover/low speed flight conditions by using scaled lateral reposition and longitudinal depart/abort mission task elements.

\section{Simulation and Flight Test Results}

Control system design and robustness analysis are followed by Monte-Carlo simulations and flight tests to evaluate and verify the closed-loop system performance. Required improvements could be defined and applied according to the obtained results. In ADS-33E-PRF, mission task elements (MTEs) are used to evaluate the system capability in good visual environments (GVE) and degraded visual environment (DVE). However, these MTEs are not suitable for the unmanned and micro aerial vehicles. So, they should be scaled down before applying to the unmanned systems.

In this section, kinematic scaling is applied to the lateral reposition and longitudinal depart/abort MTEs from ADS-33E-PRF. Then, hover and trajectory tracking and aggressiveness (TTA) analysis are performed on the technology demonstrator quadrotor platform to evaluate the proposed system performance. 


\section{A. Kinematic Scaling}

For the MTE scaling purpose, kinematic scaling is utilized successfully based on maximum forward flight speed of the aerial vehicles [17]. Maximum airspeed values of the agile maneuvering quadrotor and UH-60 are $V_{\text {max }_{\text {uav }}}=32 \mathrm{~m} / \mathrm{s}, V_{\text {max }_{\text {uh } 60}}=82.31 \mathrm{~m} / \mathrm{s}$, respectively. Spatial $\left(L_{\text {scale }}\right)$, velocity $\left(V_{\text {scale }}\right)$ and time $\left(t_{\text {scale }}\right)$ scale factors are given in Eq. (30), (31) and (32).

$$
\begin{gathered}
L_{\text {scale }}=\left(\frac{V_{\text {max }_{\text {uav }}}}{V_{\text {max }_{\text {uh } 60}}}\right)^{2} \\
V_{\text {scale }}=\alpha \frac{V_{\text {max }_{\text {uav }}}}{V_{\text {max }_{\text {uh } 60}}} \\
t_{\text {scale }}=\alpha^{-1} \frac{V_{\text {max }_{\text {uav }}}}{V_{\text {max }_{\text {uh } 60}}}
\end{gathered}
$$

where, $\alpha$ is aggressiveness level which is selected as 1,1.5,2 in this study. For more information about the MTE scaling, readers may refer to [17].

\section{B. Position-Hold Performance Evaluation in Hover Flight}

One of the most critical missions for a rotary-wing aerial vehicle is position hold flight in which adequate disturbance rejection performance is necessary. Especially in the urban environment, it is crucial to hold the commanded position with the acceptable error and overshoot to minimize the crash risk in the airspace.

To evaluate the position hold performance of the legacy and optimized controllers, hover flight tests are performed on the quadrotor platform. In these tests, given reference velocity and position commands are set to zero to maintain the hover conditions. Wind velocity is about $2-3 \mathrm{~m} / \mathrm{s}$ from North and light turbulence level is observed. Position of the quadrotor with the legacy and optimized controllers are compared in Fig. 14a. Velocity and position time histories of the legacy and optimized controllers in hover flight are given in Fig. 14b.

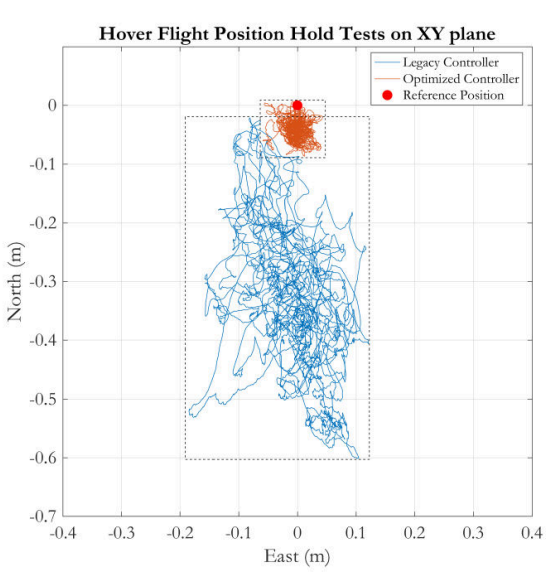

(a)
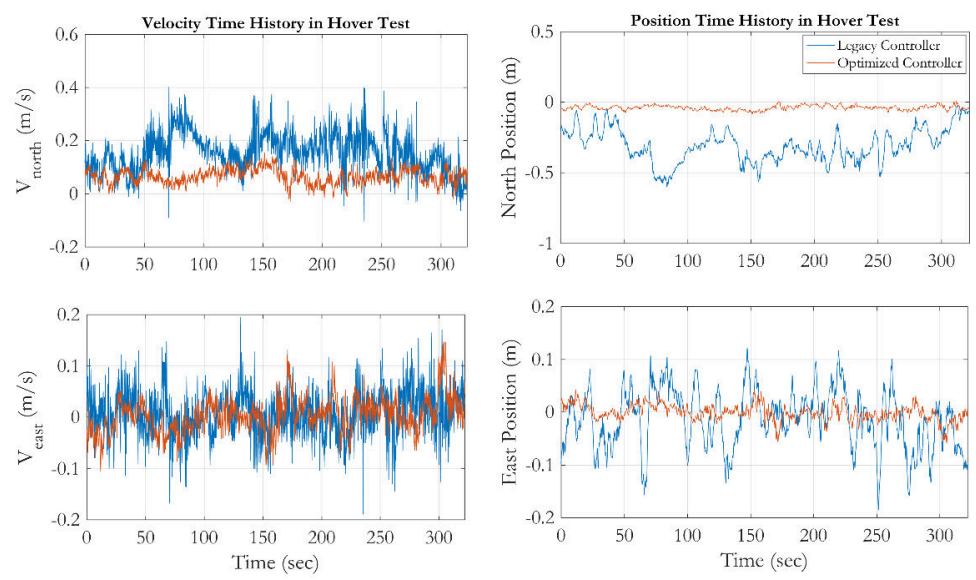

(b)

Fig. 14 a) Position-hold flight test results with the legacy and optimized controllers. b) Position and velocity time histories in hover flight test with the legacy and optimized controllers.

In these flight tests, position and velocity root-mean-square error (RMSE) of the legacy and optimized controllers are given in Table 9. As shown from the hover flight test results, the proposed controller has superior position-hold performance in the same wind conditions which is crucial for the urban environment missions.

\section{Trajectory Tracking and Aggressiveness Analysis}

In ADS-33E-PRF, there are several moderate amplitude maneuvers to evaluate the agility of the full-scale rotorcrafts. However, direct usage of these maneuvers is not sufficient for the quadrotor platforms. Hence, in the previous subsection, 
Table 9 Position and velocity RMSE for hover flight test.

\begin{tabular}{ccc}
\hline Controller & Position RMSE $(\mathrm{m})$ & Velocity RMSE $(\mathrm{m} / \mathrm{s})$ \\
\hline Legacy & 0.3364 & 0.1761 \\
Optimized & 0.0468 & 0.0791 \\
\hline
\end{tabular}

kinematic scaling with three levels of aggressiveness ( $a=1, a=1.5, a=2)$ is applied based on maximum airspeed of the quadrotor and UH-60 rotorcraft [17]. These maneuvers are performed in the stitched quasi-nonlinear model and flight tests. Velocity and position time histories are compared in Fig. 15 and 16.
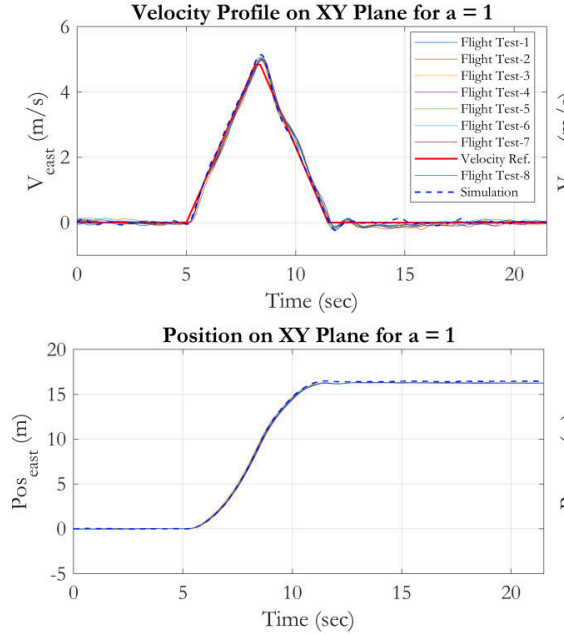
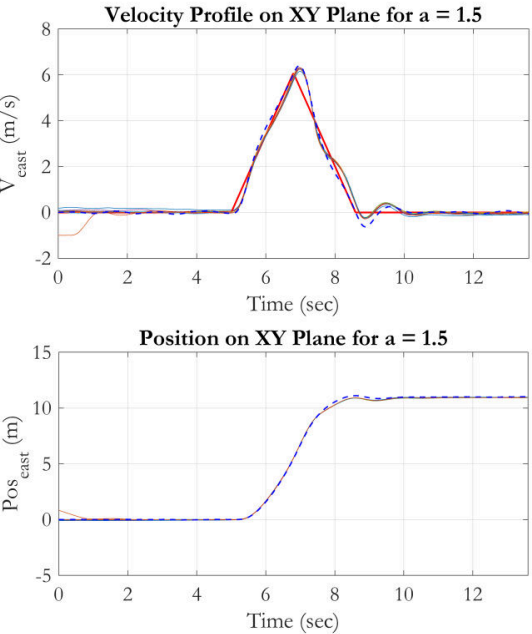
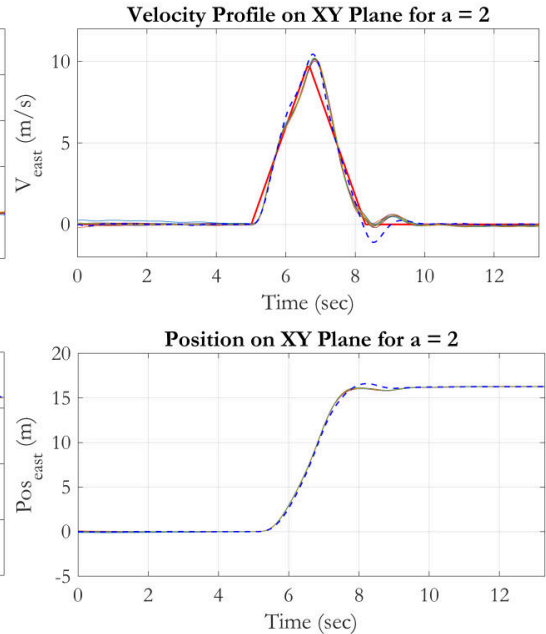

Fig. 15 Lateral reposition simulation and flight test results.
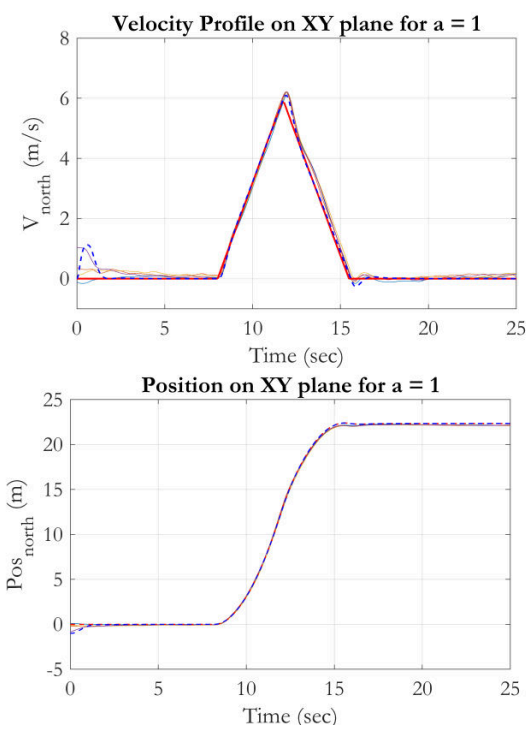
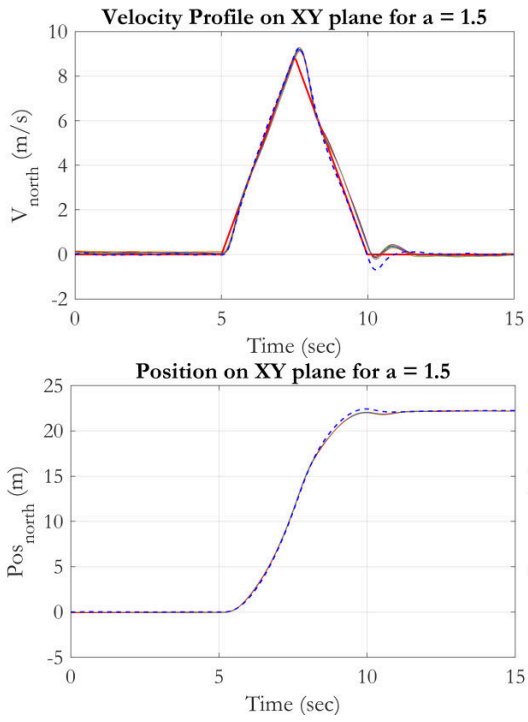
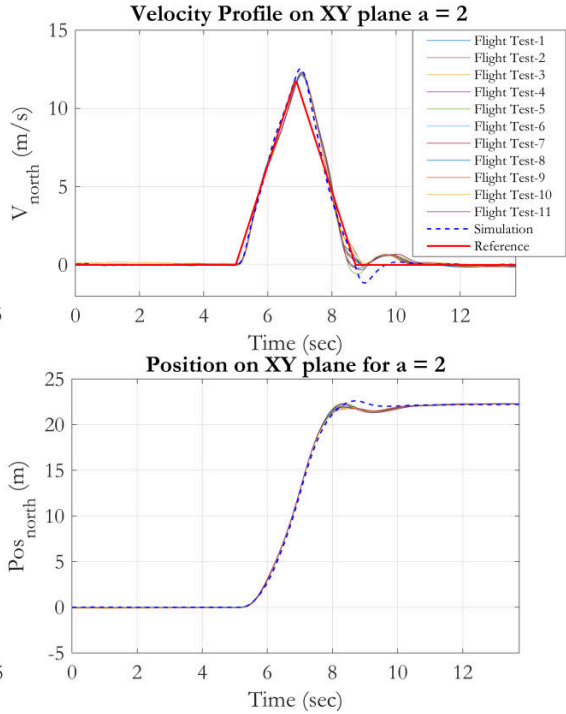

Fig. 16 Longitudinal depart/abort simulation and flight test results.

After performing several flight tests and simulations, TTA performance of the proposed closed-loop system should be evaluated quantitatively. Hence, it is required to define a cost function $(L)$ and TTA performance score $\left(\phi_{T T A}\right)$ which includes aggressiveness level, velocity and position reference tracking RMSE [17]. 
Table 10 Weights used in the TTA analysis.

\begin{tabular}{cccccccccc}
\hline Parameter & $w_{a}$ & $w_{\epsilon}$ & $w_{R}$ & $a_{G}$ & $a_{B}$ & $\epsilon_{G}$ & $\epsilon_{B}$ & $w_{\text {vel }}$ & $w_{\text {pos }}$ \\
\hline Value & 0.5 & 0.5 & 0 & 3 & 0 & 0 & 0.35 & 0.7 & 0.3 \\
\hline
\end{tabular}

Table 11 TTA score guideline for scaled lateral reposition and depart/abort MTEs [17].

\begin{tabular}{ccc}
\hline Maneuver & $\begin{array}{c}\text { Desired TTA Score } \\
\text { (Level-1) }\end{array}$ & $\begin{array}{c}\text { Adequate TTA Score } \\
\text { (Level-2) }\end{array}$ \\
\hline Lateral & $\phi_{T T A} \geq 82$ & $77 \leq \phi_{T T A} \geq 82$ \\
Reposition & $\phi_{T T A} \geq 82$ & $77 \leq \phi_{T T A} \geq 82$ \\
Depart/Abort & \\
\hline
\end{tabular}

$$
L=w_{a} \frac{a-a_{G}}{a_{B}-a_{G}}+w_{\epsilon} \frac{\epsilon-\epsilon_{G}}{\epsilon_{B}-\epsilon_{G}}+w_{R} \frac{R-R_{G}}{R_{B}-R_{G}}
$$

Here, $a$ is aggressiveness level, $\epsilon$ is tracking error term for position and velocity and $R$ is robustness of the closed-loop system which is a metric for the mission success. Subscripts ' $G$ ' and ' $B$ ' stand for the bad and good possible values for these metrics. $w_{a}, w_{\epsilon}, w_{R}$ are used to define the weights of these metrics in the cost function.

The aggressiveness term $a$ is calculated by using Eq. (34).

$$
a=\frac{V_{\text {max }_{c m d}}}{V_{\text {max }_{\text {nom }}}}
$$

where, $V_{\text {max }}$ is commanded maximum velocity and $V_{\text {max }}$ is is nominal velocity of the rotorcraft which is $5.88 \mathrm{~m} / \mathrm{s}$ for the drone platform. Velocity and position reference tracking error term in the cost function is calculated by using Eq. (35).

$$
\epsilon_{1}=w_{\text {vel }} \frac{R M S E_{v e l}}{V_{\text {max }_{c m d}}}+w_{\text {pos }} \frac{R M S E_{\text {pos }}}{L_{\text {path }}}
$$

where, $w_{v e l}, w_{\text {pos }}$ are weights for the position and velocity tracking errors. In this definition, velocity and position RMSE are normalized by using the maximum commanded velocity and length of the commanded path $L_{\text {path }}$.

In the Monte-Carlo simulations and flight tests, all of the MTEs are completed successfully. Hence, as described in [17], robustness term is not used in the cost function. However, in future studies, concept of "mission success" will be defined and robustness term will be included in the TTA analysis. In this study, the weights in the cost function are used as shown in Table 10.

After obtaining the cost function value, it is used in the TTA scoring step which is defined in Eq. (36). The value of the TTA score $\left(\phi_{T T A}\right)$ is in $0-100$ interval. High TTA score means lower tracking error and higher aggressiveness; low TTA score means higher tracking error and lower aggressiveness.

$$
\phi_{T T A}=\frac{200}{1+e^{L}}
$$

After several flight tests and Monte-Carlo simulations, TTA scores of the quadrotor platform are given in Fig. 17. Here, it is shown that the TTA scores for each aggressiveness level are above the recommended boundaries given in Table 11 [17]. Also, the optimized controller has similar TTA scores in Monte-Carlo simulation and flight tests which validates the mathematical model and control systems.

The legacy controller parameters are not suitable for this type of trajectory tracking control system structure. Oscillatory responses are observed in flight tests in pitch and roll axes. Because of the safety considerations, TTA scoring analysis of the closed-loop system with the legacy controller is not performed. 


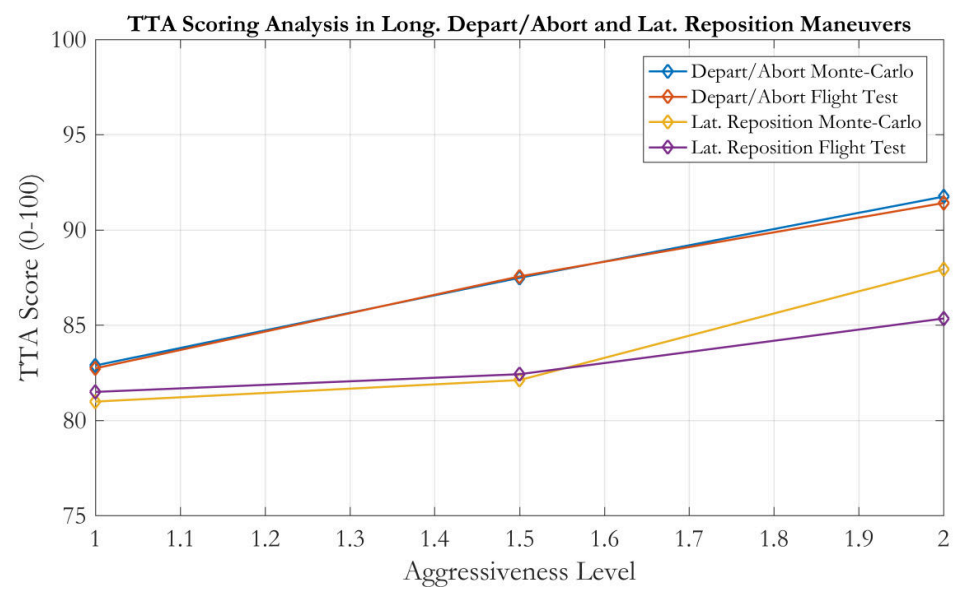

Fig. 17 TTA Scoring of the depart/abort and lateral reposition maneuvers with optimized controllers.

\section{Conclusion}

Desktop-to-flight design workflow is applied on an highly agile quadrotor platform to obtain suitable inner and outerloop controllers for high precision and agile trajectory tracking missions. The frequency-domain system identification process is used in both hover/low speed and fast forward flight phases to identify the bare-airframe dynamics. Then, obtained point models are stitched and quasi-nonlinear simulation environment is generated. Inner and outer-loop legacy controllers are modified for the trajectory tracking mission and optimized controller parameters are determined by using multi-objective optimization based controller design process. The trajectory tracking performances of the legacy and optimized control systems are evaluated by utilizing Monte-Carlo simulations and outdoor flight tests.

The results indicate that, similar to the dynamical behaviour of a full-scale rotorcraft, there are significant deviations in bare-airframe dynamics of the quadrotor platform in hover and forward flight conditions. In comparison to classical control designs, the optimized controllers (across hover/low speed and high forward speed flight conditions) show significant precision, predictability and robustness.

Current work focuses on further improvement of the reference signal tracking performance of the racer quadrotor platform in higher speed $(20-32 \mathrm{~m} / \mathrm{s})$ forward flight conditions.

\section{Appendix}
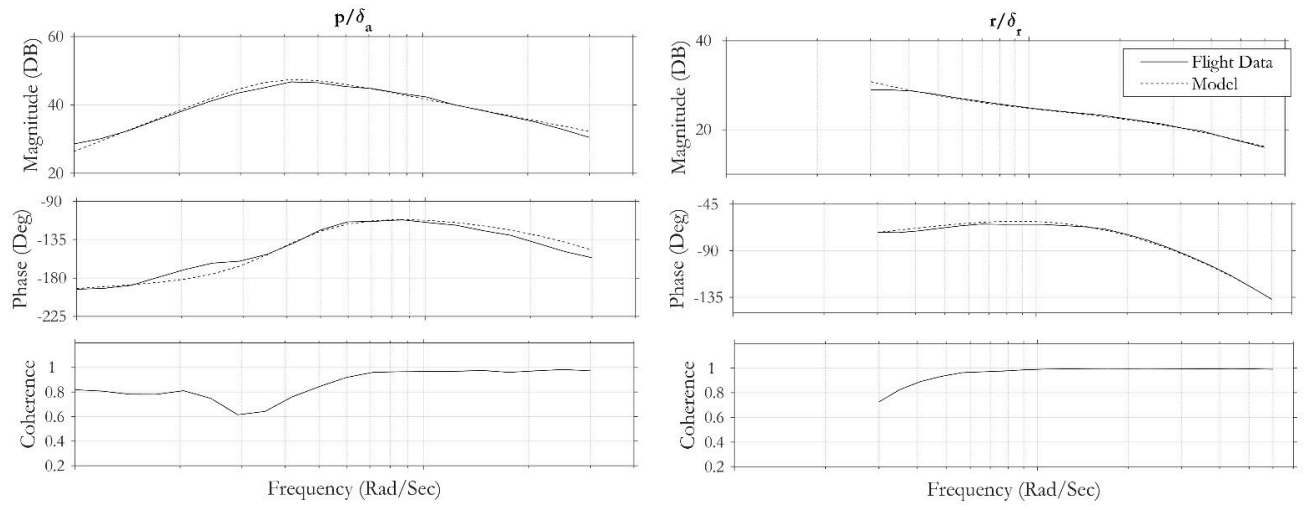

Fig. 18 On-Axis $p / \delta_{a}, r / \delta_{r}$ frequency responses of the actual system and identified model in hover flight conditions. 

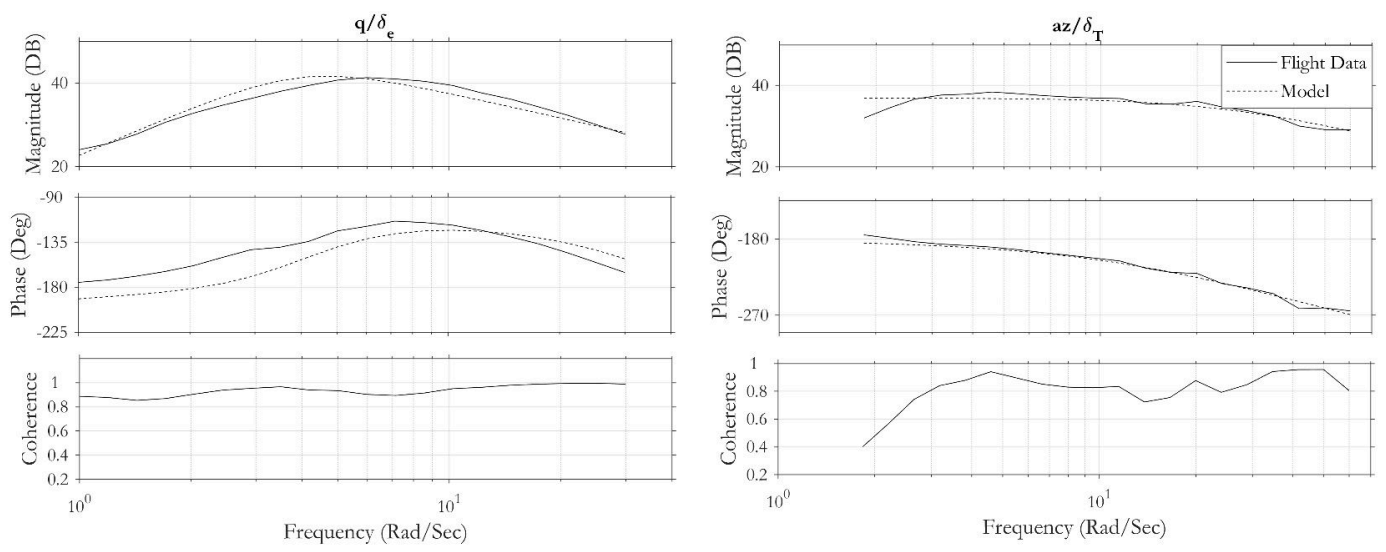

Fig. 19 On-Axis $q / \delta_{e}, a_{z} / \delta_{T}$ frequency responses of the actual system and identified model in hover flight conditions.
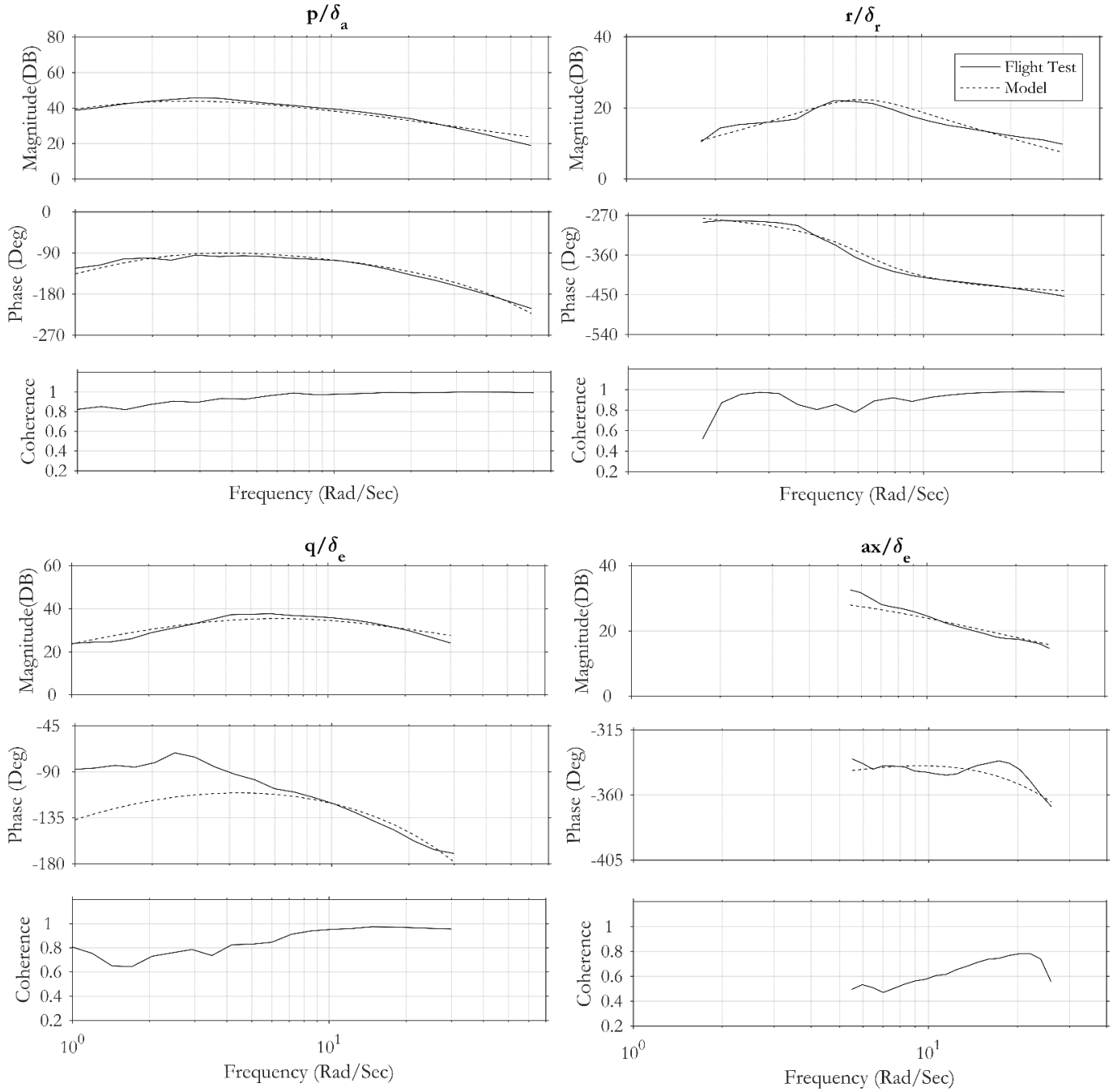

Fig. 20 On-Axis frequency responses of the actual system and identified model in forward flight conditions. 

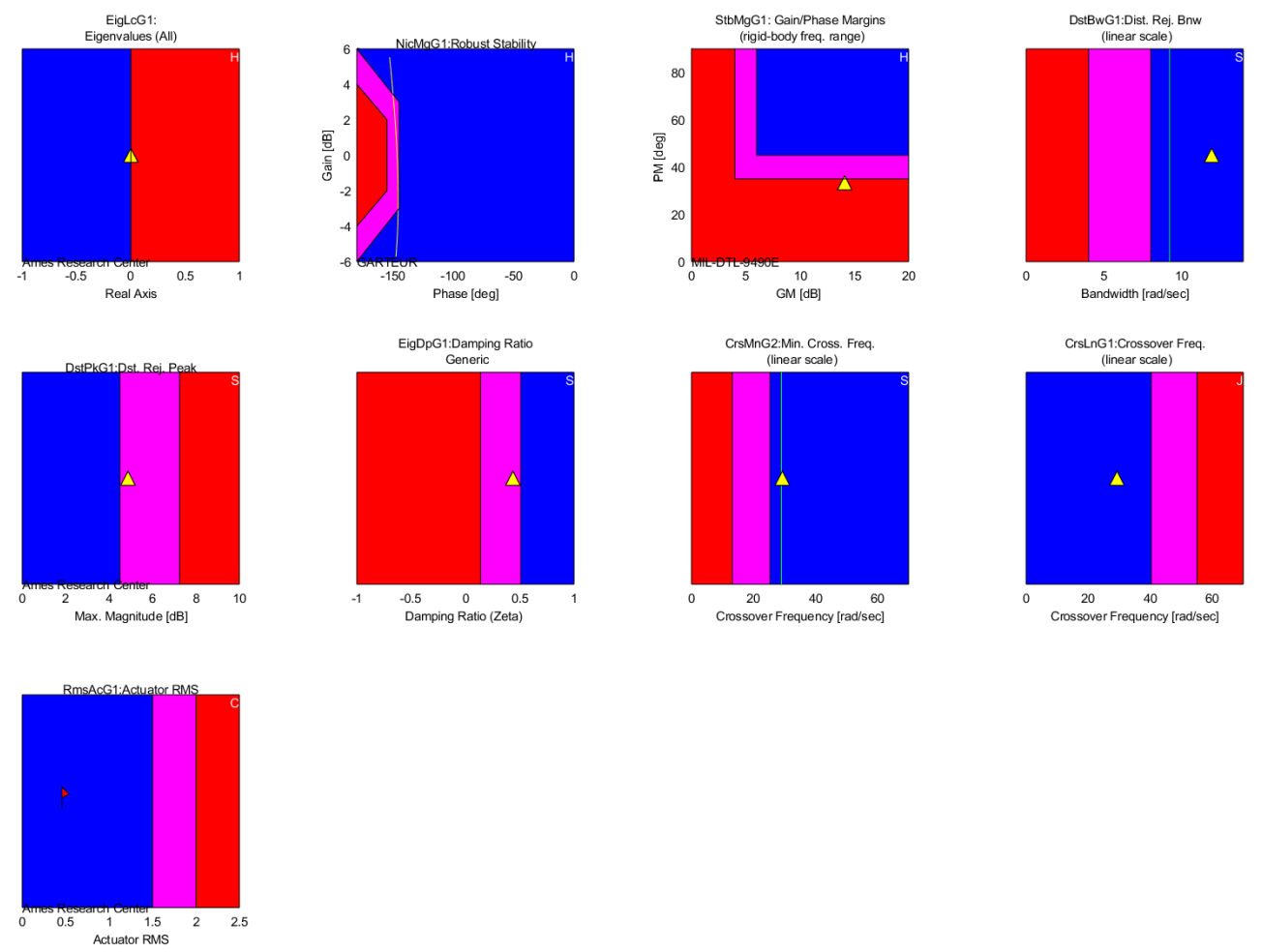

Fig. 21 Performance evaluation of ArduCopter roll attitude controller in CONDUIT for hover/low speed conditions.

\section{References}

[1] Tischler, M. B., Berger, T., Ivler, C. M., Mansur, M. H., Cheung, K. K., and Soong, J. Y., "Practical Methods for Aircraft and Rotorcraft Flight Control Design: An Optimization-Based Approach,” AIAA education series, 2017.

[2] Anon., “Military Standard, Flying Qualities of Piloted Airplanes, MIL-STD-1797B,” Tech. rep., 2012.

[3] Anon., "Aeronautical Design Standard, Performance Specification, Handling Qualities Requirements for Military Rotorcraft, ADS-33E-PRF," Tech. rep., US Army Aviation and Missile Command, 2000.

[4] Sanders, F. C., Tischler, M., Berger, T., Berrios, M. G., and Gong, A., "System Identification and Multi-Objective Longitudinal Control Law Design for a Small Fixed-Wing UAV," 2018 AIAA Atmospheric Flight Mechanics Conference, 2018 , p. 0296.

[5] Yuksek, B., Saldiran, E., Cetin, A., Yeniceri, R., and Inalhan, G., "A Model Based Flight Control System Design Approach for Micro Aerial Vehicle Using Integrated Flight Testing and HIL Simulation,” AIAA Scitech 2019 Forum, 2019, p. 1480.

[6] Wei, W., Cohen, K., and Tischler, M. B., "System identification and controller optimization of a quadrotor UAV," Proceedings of the AHS International's 71st Annual Forum and Technology Display, Virginia, USA, 2015.

[7] Juhasz, T. M., Ondrej, and Won, H., "System Identification and Control Law Optimization Applied to the AeroVironment Quantix Tail-Sitter UAS," Proceedings of the AHS International's 74st Annual Forum and Technology Display, Arizona, USA, 2018.

[8] Zivan, L., and Tischler, M. B., "Development of a full flight envelope helicopter simulation using system identification,” Journal of the American Helicopter Society, Vol. 55, No. 2, 2010, pp. 22003-22003.

[9] Knapp, M. E., Berger, T., Tischler, M., and Cotting, M. C., "Development of a Full Envelope Flight Identified F-16 Simulation Model," 2018 AIAA Atmospheric Flight Mechanics Conference, 2018, p. 0525.

[10] Mansur, M. H., Tischler, M. B., Bielefield, M. D., Bacon, J. W., Cheung, K. K., Berrios, M. G., and Rothman, K. E., "Full Flight Envelope Inner Loop Control Law Development for the Unmanned K-MAX,” Tech. rep., 2011. 

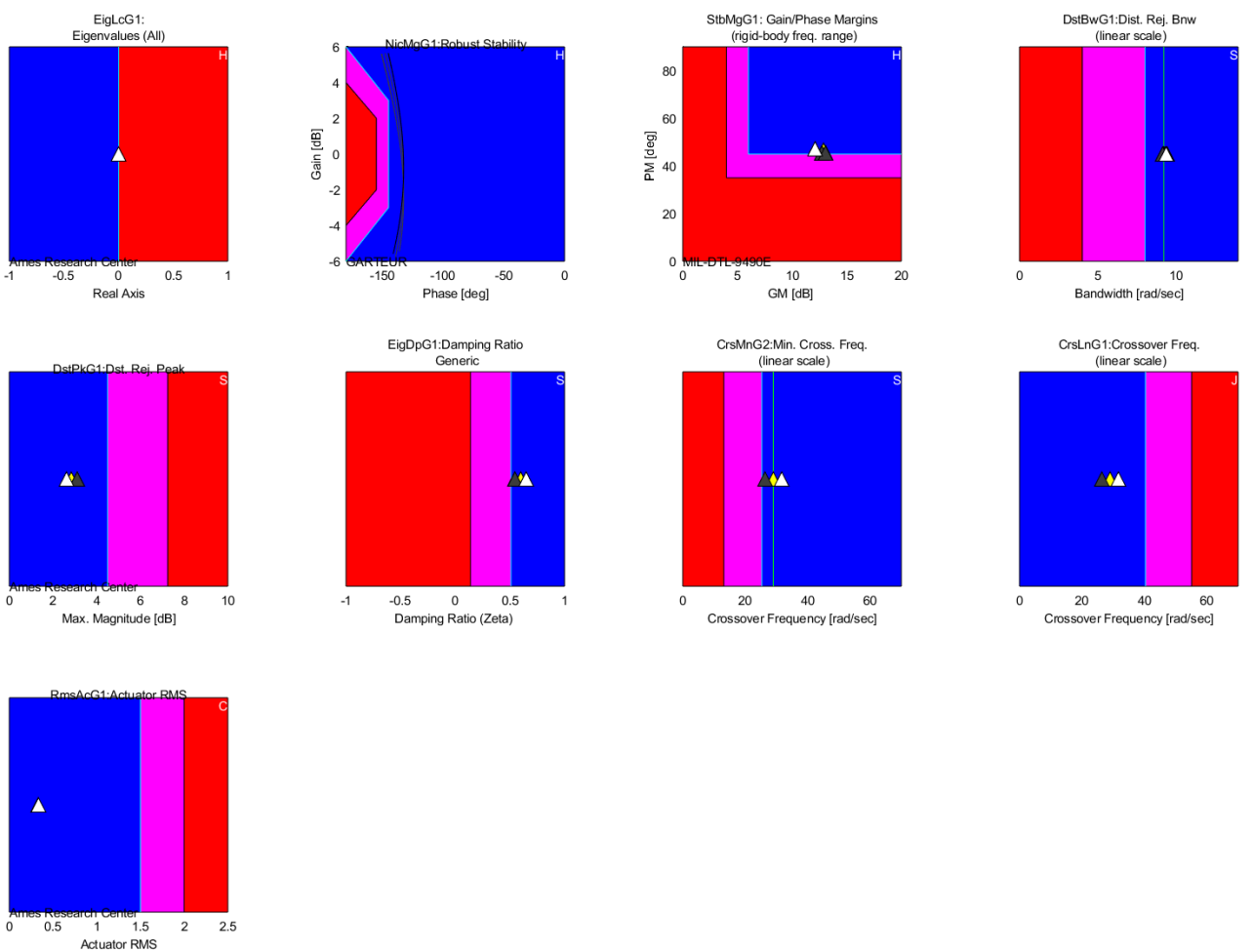

Fig. $223 \sigma$ robustness analysis of optimized roll attitude controller in CONDUIT for hover/low speed conditions.

[11] Tobias, E., Sanders, F., and Tischler, M. B., "Full-Envelope Stitched Simulation Model of a Quadrotor using STITCH," American Helicopter Society 74th Annual Forum, 2018.

[12] Gong, A., Sanders, F. C., Hess, R. A., and Tischler, M. B., "System Identification and Full Flight-Envelope Model Stitching of a Package-Delivery Octocopter," AIAA Scitech 2019 Forum, 2019, p. 1076.

[13] Tischler, M. B., and Remple, R. K., “Aircraft and Rotorcraft System Identification,” AIAA education series, 2012.

[14] Cheung, K. K., Wagster IV, J. A., Tischler, M. B., Ivler, C. M., Berrios, M. G., Berger, T., and Lehmann, R. M., "An Overview of the US Army Aviation Development Directorate Quadrotor Guidance, Navigation, and Control Project," Vertical Flight Society Forum, Vol. 73, 2017.

[15] Tischler, M. B., and Tobias, E. L., "A Model Stitching Architecture for Continuous Full Flight-Envelope Simulation of FixedWing Aircraft and Rotorcraft from Discrete Point Linear Models," Tech. rep., Aviation and Missile Research, Development and Engineering Center, Redstone, 2016.

[16] Arducopter, (accessed May 26, 2019). URL http://ardupilot.org/copter/.

[17] Ivler, C. M., and Goerzen, C. L., "Control Design for Tracking of Scaled MTE Trajectories on an IRIS+ Quadcopter," American Helicopter Society International 74th Annual Forum and Technology Display, Phoenix, Arizona, USA, May 14-17, 2018.

[18] Berger, T., Ivler, C. M., Berrios, M. G., Tischler, M. B., and Miller, D., "Disturbance Rejection Handling Qualities Criteria for Rotorcraft," AHS 72nd Annual Forum, West Palm Beach, FL, 2016. 
2020-01-05

System identification and model-based

flight control system design for an agile maneuvring quadrotor platform

Yuksek, Burak

AIAA

Yuksek B, Saldiran E, Cetin A, et al., (2020) System identification and model-based flight control system design for an agile maneuvring quadrotor platform. In: 2020 AIAA SciTech Forum, Orlando, Florida, 6-10 January 2020

https://doi.org/10.2514/6.2020-1835

Downloaded from Cranfield Library Services E-Repository 